\title{
Coefficient multipliers on Banach spaces of analytic functions
}

\section{Óscar Blasco and Miroslav Pavlović}

\begin{abstract}
Motivated by an old paper of Wells [34] we define the space $X \otimes Y$, where $X$ and $Y$ are "homogeneous" Banach spaces of analytic functions on the unit disk $\mathbb{D}$, by the requirement that $f$ can be represented as $f=\sum_{j=0}^{\infty} g_{n} * h_{n}$, with $g_{n} \in X, h_{n} \in Y$ and $\sum_{n=1}^{\infty}\left\|g_{n}\right\|_{X}\left\|h_{n}\right\|_{Y}<\infty$. We show that this construction is closely related to coefficient multipliers. For example, we prove the formula $((X \otimes Y), Z)=(X,(Y, Z))$, where $(U, V)$ denotes the space of multipliers from $U$ to $V$, and as a special case $(X \otimes Y)^{*}=\left(X, Y^{*}\right)$, where $U^{*}=\left(U, H^{\infty}\right)$. We determine $H^{1} \otimes X$ for a class of spaces that contains $H^{p}$ and $\ell^{p}(1 \leq p \leq 2)$, and use this together with the above formulas to give quick proofs of some important results on multipliers due to Hardy and Littlewood, Zygmund and Stein, and others.
\end{abstract}

\section{Introduction}

Let $\mathcal{S}$ denote the space of all (formal) power series $f=\sum_{j=0}^{\infty} \hat{f}(j) z^{j}=$ $\{\hat{f}(j)\}_{j=0}^{\infty}$ with complex-valued coefficients. We introduce the locally convex vector topology on $X$ by means of the seminorms $p_{j}(f)=\hat{f}(j), j \geq 0$. Thus $f_{n} \rightarrow f(n \rightarrow \infty)$ in $\mathcal{S}$ if and only if $\hat{f}_{n}(j) \rightarrow \hat{f}(j)$ for each $j$. Then $\mathcal{S}$ is metrizable and complete and therefore it is an $F$-space. The Hadamard product of $f$ and $g$ is defined as

$$
f * g=\sum_{j=0}^{\infty} \hat{f}(j) \hat{g}(j) z^{j} .
$$

A Banach space $X$ will be called $\mathcal{S}$-admissible if $\mathcal{P}$, the set of all polynomials, is contained in $X$, and $X \subset \mathcal{S}$ with continuous inclusion.

2000 Mathematics Subject Classification: Primary: 42A45, 30A99; Secondary: 30D55, 46E15, 46A45.

Keywords: Banach spaces, analytic functions, coefficient multipliers, tensor products, Hardy spaces. 
Let $X_{\mathcal{P}}$ denote the closure of $\mathcal{P}$ in $X, e_{j}(z)=z^{j}$ and $\gamma_{j}(f)=\hat{f}(j)$ for $j \geq 0$. Of course if $X$ is $\mathcal{S}$-admissible so it is $X_{\mathcal{P}}$. On the other hand for an $\mathcal{S}$-admissible Banach space $X$ one has that $e_{j} \in X$ and $\gamma_{j} \in X^{\prime}$, where $X^{\prime}$ stands for the topological dual space. Hence $\left(X_{\mathcal{P}}\right)^{\prime}$ is also an $\mathcal{S}$-admissible Banach space, identifying $\phi \in X^{\prime}$ with the power series $\phi(z)=\sum_{j} \phi\left(e_{j}\right) z^{j}$.

Note that $\ell^{p}, 1 \leq p \leq \infty$, the space of all complex sequences $a=\{\hat{a}(j)\}_{0}^{\infty}$ such that $\|a\|_{\ell^{p}}:=\left(\sum_{j=0}^{\infty}|\hat{a}(j)|^{p}\right)^{1 / p}<\infty$, can be regarded as a subspace of $\mathcal{S}$, denoted $A(\mathbb{T})$ for $p=1$, by putting $a=\sum_{j=0}^{\infty} \hat{a}(j) z^{j}$. Further examples of $\mathcal{S}$-admissible spaces are $c_{0}=\left(\ell^{\infty}\right)_{\mathcal{P}}, H^{\infty}$, i.e. the space of bounded analytic functions and $A(\mathbb{D})=\left(H^{\infty}\right)_{\mathcal{P}}$.

Given two $\mathcal{S}$-admissible Banach spaces $X, Y$ we denote

$$
(X, Y)=\{\lambda \in \mathcal{S}: \lambda * f \in Y \text { for all } f \in X\} .
$$

Then $(X, Y)$ becomes an $\mathcal{S}$-admissible Banach space with its natural norm (see Theorem 2.1).

We keep the notation $X^{\prime}$ for the topological dual and denote $X^{K}=$ $(X, A(\mathbb{T}))$ (the Köthe dual), $X^{*}=\left(X, H^{\infty}\right)$ and $X^{\#}=(X, A(\mathbb{D}))$.

Since $H^{\infty}, A(\mathbb{T}), A(\mathbb{D})$ are $\mathcal{S}$-admissible Banach spaces then $X^{K}, X^{*}$ and $X^{\#}$ are also $\mathcal{S}$-admissible Banach spaces.

Following Wells [34] (see also [11] and [33, Sections V.4, VI.3]), given $X$ and $Y \mathcal{S}$-admissible Banach spaces we define $X \otimes Y$ as the space of series $h \in \mathcal{S}$ such that $h=\sum_{n=0}^{\infty} f_{n} * g_{n}$, where the series converges in $\mathcal{S}, f_{n} \in X$, $g_{n} \in Y$ and $\sum_{n=0}^{\infty}\left\|f_{n}\right\|_{X}\left\|g_{n}\right\|_{Y}<\infty$. It is not difficult to see that $X \otimes Y$, normed in a natural way, is also $\mathcal{S}$-admissible (see Theorem 2.2).

We shall show in the paper a quite useful formula connecting multipliers and tensors of $\mathcal{S}$-admissible Banach spaces (see Theorem 2.3)

$$
(X \otimes Y, Z)=(X,(Y, Z))
$$

We are mainly interested in the case where $X$ and $Y$ are Banach spaces of analytic functions on the unit disk $\mathbb{D} \subset \mathbb{C}$, i.e., $f=\sum \hat{f}(j) z^{j}$ with $\limsup \operatorname{suj}_{j} \sqrt[j]{|\hat{f}(j)|} \leq 1$. Let $\mathbb{D}_{R} \subset \mathbb{C}$ denote the open disk of radius $R$ centered at zero (we put $\mathbb{D}_{1}=\mathbb{D}$ ) and let $E$ be a complex Banach space. We write $\mathcal{H}\left(\mathbb{D}_{R}\right)$ (respect. $\mathcal{H}\left(\mathbb{D}_{R}, E\right)$ ) for the vector space of all functions analytic in $\mathbb{D}_{R}$ (respect. with values in $E$ ), which endowed with "H-H-topology", i.e., the topology of uniform convergence on compact subsets of $\mathbb{D}_{R}$, becomes a locally convex $F$-space. This topology can be described by the family of the norms $N_{\rho}(f)=\sup _{|z|<\rho}\|f(z)\|_{E}, 0<\rho<R$. Since $\mathcal{H}\left(\mathbb{D}_{R}\right) \subset \mathcal{S}$, we see that, formally, there are two topologies on $\mathcal{H}\left(\mathbb{D}_{R}\right): \mathcal{H}$-topology and $\mathcal{S}$-topology. However, it is well known and easy to see that they coincide on $\mathcal{H}\left(\mathbb{D}_{R}\right)$. 
Several authors have formulated some natural conditions (which hold in most of classical spaces such as Hardy, Bergman, Besov, etc.) to develop a general theory of spaces of analytic functions. Two basic ones first appeared in the work by A.E. Taylor (see [29]) are the following:

(P1) There exists $A_{1}>0$ such that $|\hat{f}(j)| \leq A_{1}\|f\|, j \in\{0,1, \ldots\}$.

(P2) There exists $A_{2}>0$ such that $\left\|e_{j}\right\| \leq A_{2}, j \in\{0,1, \ldots\}$.

This perfectly fitted with Hardy spaces (see [30]) but, unfortunately these conditions are too restrictive to include many of the interesting spaces appearing in the literature. We shall propose in this paper some weaker ones.

A Banach space $X \subset \mathcal{S}$ will be called $\mathcal{H}$-admissible if $X \subset \mathcal{H}(\mathbb{D})$ with continuous inclusion, $\mathcal{H}\left(\mathbb{D}_{R}\right) \subset X$ for all $R>1$, and the map $\left.f \mapsto f\right|_{\mathbb{D}}$ is continuous from $\mathcal{H}\left(\mathbb{D}_{R}\right)$ to $X$.

Clearly $\mathcal{H}$-admissible spaces are also $\mathcal{S}$-admissible. Denote, as usual, $C(z)=\frac{1}{1-z}$ the Cauchy kernel and $f_{w}(z)=f(w z)$ for $w \in \overline{\mathbb{D}}$. In particular $f_{r}=C_{r} * f$.

We shall show that in the setting of $\mathcal{H}$-admissible Banach spaces, the map $w \rightarrow f_{w}$ defines an $X$-valued analytic function, i.e. $F(w)=f_{w} \in \mathcal{H}(\mathbb{D}, X)$. In particular

$$
M_{X}(r, f)=\sup _{|w|=r}\left\|f_{w}\right\|_{X}
$$

becomes an increasing function (where, as usual, we denote $M_{p}(r, f)$ for the Hardy spaces $X=H^{p}$ ). We shall pay special attention to the subspace of functions such that $F \in H^{\infty}(\mathbb{D}, X)$ and denote

$$
\tilde{X}=\left\{f \in \mathcal{H}(\mathbb{D}): \sup _{0<r<1} M_{X}(r, f)<\infty\right\} .
$$

Of course if $X$ and $Y$ are $\mathcal{H}$-admissible then $(X, Y)$ and $X \otimes Y$ are also $\mathcal{H}$-admissible (see Theorem 3.1).

Inspired by the Besov-type spaces we denote, for $1 \leq q \leq \infty$, by $\mathfrak{B}^{X, q}$ the space of functions in $\mathcal{H}(\mathbb{D})$ such that $\left(1-r^{2}\right) M_{X}(r, D f) \in L^{q}\left((0,1), \frac{r d r}{1-r^{2}}\right)$ where $D f(z)=\sum_{n=0}^{\infty}(n+1) \hat{f}(n) z^{n}$.

It is clear that $\tilde{X}$ and $\mathfrak{B}^{X, q}$ are also $\mathcal{H}$-admissible Banach spaces. It fact they automatically have better properties.

In the original paper A.E. Taylor also considered some particular properties (see [29]):

(P3) If $f \in X$ then $f_{e^{i \theta}} \in X$ and $\left\|f_{e^{i \theta}}\right\|_{X}=\|f\|_{X}, \theta \in[0,2 \pi]$.

(P4) If $f \in X$ then $f_{r} \in X$ with $\left\|f_{r}\right\|_{X} \leq A_{4}\|f\|_{X}, 0 \leq r<1$, for some $A_{4}>0$.

In this paper we propose a general class of $\mathcal{H}$-admissible Banach spaces of analytic functions, which cover many of the classical function spaces, and is well-adapted to the study of multipliers. 
We shall say that an $\mathcal{H}$-admissible Banach space $X$ is homogeneous if (P3) and (P4) holds, that is, it satisfies $\left\|f_{\xi}\right\|_{X}=\|f\|_{X}$ for all $|\xi|=1$ and $f \in X$, and $M_{X}(r, f) \leq K\|f\|_{X}$ for all $0 \leq r<1$ and $f \in X$.

That is to say, for homogeneous spaces, $w \rightarrow f_{w}$ defines a function in $H^{\infty}(\mathbb{D}, X)$. In particular $X \subset \tilde{X}$.

Note that the spaces $\tilde{X}$ and $\mathfrak{B}^{X, q}$ become automatically homogeneous for any $\mathcal{H}$-admissible Banach space $X$. Of course if $X$ and $Y$ are homogeneous so are $(X, Y)$ and $X \otimes Y$.

We shall also show in this setting that (see Theorem 7.1)

$$
\mathfrak{B}^{X, 1} \subset H^{1} \otimes X \subset X_{\mathcal{P}}
$$

or that (see Theorem 4.1)

$$
\left(\mathfrak{B}^{X, 1}, Y\right)=\mathfrak{B}^{(X, Y), \infty} .
$$

Many more properties are relevant according to the problem in study. For instance, the class of spaces invariant under Moebious transformations or $G$-invariant spaces, i.e. $X \subset \mathcal{H}(\mathbb{D})$ such that there exists $K>0$ such that $\|f \circ \phi\|_{X} \leq K\|f\|_{X}$ whenever $f \in X$ and $\phi$ belongs to the group of Moebious transformation of $\mathbb{D}$, have been considered by several authors (see [3, 12, 31]). Among the $G$-invariant spaces there are maximal and minimal spaces in the scale, namely the Bloch space and the Besov class (see [6, 26, 32]). Similarly, in our setting of homogeneous Banach spaces of analytic functions one has (see Proposition 4.3) that

$$
\mathfrak{B}^{X, 1} \subset X_{\mathcal{P}} \subset \tilde{X} \subset \mathfrak{B}^{X, \infty} .
$$

Let us finally recall some extra properties also considered by Taylor:

(P5) If $f \in X$ then $f_{r} \in X$ and $\|f\|_{X}=\lim _{r \rightarrow 1}\left\|f_{r}\right\|_{X}$.

(P6) If $f \in X$ then $f_{r} \in X$ and $\lim _{r \rightarrow 1}\left\|f_{r}-f\right\|_{X}=0$.

Of course these two conditions are connected to the density of polynomial in the space $X$. In fact if $X$ is $\mathcal{H}$-admissible then $X_{\mathcal{P}}$ satisfies (P6) (and therefore (P5)).

Another one which appears naturally is the following:

(P7) If $f \in \mathcal{H}(\mathbb{D})$ satisfies that $f_{r} \in X$ and $\sup _{r \rightarrow 1}\left\|f_{r}\right\|_{X}<\infty$ then $f \in X$ and $\|f\|_{X}=\lim _{r \rightarrow 1}\left\|f_{r}\right\|_{X}$.

This is satisfied by $\tilde{X}$ and $\mathfrak{B}^{X, q}$. Clearly $c_{0}$ or $A(\mathbb{D})$ fail this property. We shall consider a variation of (P7) useful for our purposes. An homogeneous space $X$ is said to have $(F)$-property (Fatou property) if there exists $A>0$ such that for any sequence $\left(f_{n}\right) \in X$ with $\sup _{n}\left\|f_{n}\right\|_{X} \leq 1$ and $f_{n} \rightarrow f$ in $\mathcal{H}(\mathbb{D})$ one has that $f \in X$ and $\|f\|_{X} \leq A$. $(F)$-property will be shown to be equivalent to the fact that $X=\tilde{X}$ or $X=X^{* *}$ with equivalent norms (see Proposition 5.1). 
One of our main goals is to characterize $H^{1} \otimes X$. In order to do that we shall consider a new property, namely, we say that $X$ has the $(H L P)$ property if $X \subset \mathfrak{B}^{X, 2}$. For instance $\ell^{q}$ fails to have $(H L P)$ for $q>2$, because $\mathfrak{B}^{\ell^{q}, 2}=\ell(q, 2)$ (see Proposition 3.6), and $H^{p}$ has $(H L P)$ for $1 \leq p \leq 2$ due to the Hardy and Littlewood result (see [10, 15]) which states that, for $1 \leq p \leq 2$,

$$
\int_{0}^{1}\left(1-r^{2}\right) M_{p}^{2}\left(r, f^{\prime}\right) r d r \leq C\|f\|_{p}^{2}, \quad f \in H^{p} .
$$

The vector-valued version of the Hardy-Littlewood theorem was considered in [5]. A Banach space $E$ was said to have the $(H L)$-property if

$$
\int_{0}^{1}\left(1-r^{2}\right) M_{1}^{2}\left(r, F^{\prime}\right) r d r \leq C\|F\|_{H^{1}(\mathbb{D}, X)}^{2}, \quad F \in H^{1}(\mathbb{D}, E) .
$$

Since $F(w)=f_{w} \in H^{\infty}(\mathbb{D}, X)$ and $\|F\|_{H^{1}(\mathbb{D}, X)}=\|f\|_{X}$ for any $f \in X$ and any homogeneous space $X$, one concludes that any homogeneous Banach space $X$ having the $(H L)$-property satisfies $(H L P)$. The reader is referred to [5] for examples of such spaces and connections with other properties in Banach space theory. In particular it was shown ([5, Prop. 4.4]) that $L^{p}(\mu)$ has $(H L)$ if and only if $1 \leq p \leq 2$. Therefore, besides Hardy spaces, also Bergman spaces $X=A^{p}$ or $X=\ell^{p}$ for $1 \leq p \leq 2$ and many other obtained via interpolation satisfy $(H L P)$.

We shall show that if $X$ has $(H L P)$ property then (see Theorem 7.2)

$$
H^{1} \otimes X=\mathfrak{B}^{X, 1} .
$$

A combination of our main results (1.4), (1.1) and (1.3) allows us to recover a number of know results about multipliers. Namely, for spaces with $(H L P)$ one has

$$
\left(H^{1}, X^{*}\right)=(X, B M O A)=\mathfrak{B}^{X^{*}, \infty} .
$$

From this one can recapture many known results on multipliers and to obtain new ones selecting other spaces with $(H L P)$.

The paper is organized as follows: Sections 2 is devoted to introduce and prove the basic properties about the $\mathcal{S}$-admissibility showing there the basic formula (1.1). Section 3 deals with the notion of $\mathcal{H}$-admissibility. We also introduce in that section the spaces $\tilde{X}$ and $\mathfrak{B}^{X, q}$. We deal with the notion of homogeneous Banach spaces in Section 4, showing there the basic result of multipliers (1.3). The Fatou property is studied in Section 5. In Section 6 we present some new facts on "solid" spaces (introduced and studied by Anderson and Shields [2]). We use Section 7 to study the space $H^{1} \otimes X$ and to show (1.2) and (1.4). Finally Section 8 is devoted to applications. 


\section{2. $\mathcal{S}$-admissible Banach spaces: Multipliers and tensors}

Definition 2.1. A Banach space $X$ will be called $\mathcal{S}$-admissible if $\mathcal{P} \subset X$ and $X \subset \mathcal{S}$ with continuous inclusion, i.e. for each $j \geq 0$ there exists $C_{j}$ such that $|\hat{f}(j)| \leq C_{j}\|f\|_{X}$.

Definition 2.2. Let $X$ and $Y$ be $\mathcal{S}$-admissible Banach spaces. A series $\lambda \in \mathcal{S}$ is said to be a (coefficient) multiplier from $X$ to $Y$ if $\lambda * f \in Y$ for each $f \in X$.

We denote the set of all multipliers from $X$ to $Y$ by $(X, Y)$ and define

$$
\|\lambda\|_{(X, Y)}=\sup \left\{\|\lambda * f\|_{Y}:\|f\|_{X} \leq 1\right\} .
$$

Theorem 2.1. If $X$ and $Y$ are $\mathcal{S}$-admissible then $(X, Y)$ is an $\mathcal{S}$-admissible Banach space.

Proof. An application of the closed graph theorem shows that the functional $\|\cdot\|_{(X, Y)}$ is finite. That $\|\lambda\|_{(X, Y)}=0$ implies $\lambda=0$ follows the condition $\mathcal{P} \subset X$. The other properties of the norm are immediate consequences of the definition. Also, it is clear that $\mathcal{P} \subset(X, Y)$. That the inclusion $(X, Y) \subset \mathcal{S}$ is continuous follows from the inequality

$$
\left.|\hat{\lambda}(j)|=\mid \widehat{\left(\lambda * e_{j}\right.}\right)(j) \mid \leq C_{j}\left\|\lambda * e_{j}\right\|_{Y} \leq C_{j}\left\|e_{j}\right\|_{X}\|\lambda\|_{(X, Y)} .
$$

Finally, to prove that $(X, Y)$ is complete, assume that

$$
\left\|\lambda_{m}-\lambda_{n}\right\|_{(X, Y)} \rightarrow 0 \text { as } m, n \rightarrow \infty .
$$

This implies that there is a bounded linear operator $T: X \mapsto Y$ such that $\left\|T-T_{n}\right\| \rightarrow 0$ as $n \rightarrow \infty$, where the linear operator $T_{n}$ is defined by $T_{n} f=\lambda_{n} * f$. Hence $\left\|T f-\lambda_{n} * f\right\|_{Y} \rightarrow 0$ as $n \rightarrow \infty$, for each $f \in X$. Since the inclusion $Y \subset \mathcal{S}$ is continuous, we see that

$$
\lambda_{n} * f \rightarrow T f \text { in } \mathcal{S} .
$$

On the other hand, from $(+)$ and the continuity of the inclusion $(X, Y) \subset \mathcal{S}$ it follows that $\lambda_{m}-\lambda_{n} \rightarrow 0(m, n \rightarrow \infty)$ in $\mathcal{S}$, which implies that there is a $\lambda \in \mathcal{S}$ such that $\lambda_{n} * f \rightarrow \lambda * f$ in $\mathcal{S}$. This and $(*)$ show that $T f=\lambda * f$, which completes the proof.

We have another procedure to generate $\mathcal{S}$-admissible Banach spaces.

Definition 2.3. We define the space $X \otimes Y$, to be the set of all $h \in \mathcal{S}$ that can be represented in the form $h=\sum_{n=0}^{\infty} f_{n} * g_{n}, \quad f_{n} \in X, g_{n} \in Y$ so that the series converges in $\mathcal{S}$ and

$$
\sum_{n=0}^{\infty}\left\|f_{n}\right\|_{X}\left\|g_{n}\right\|_{Y}<\infty
$$


The norm in $X \otimes Y$ is given by

$$
\|h\|_{X \otimes Y}=\inf \sum_{n=0}^{\infty}\left\|f_{n}\right\|_{X}\left\|g_{n}\right\|_{Y},
$$

where the infimum is taken over all the above representations.

It follows from the definition that if (2.1) holds, then $\sum_{n=0}^{\infty} f_{n} * g_{n} \in$ $X \otimes Y$, and

$$
\left\|\sum_{n=0}^{\infty} f_{n} * g_{n}\right\|_{X \otimes Y} \leq \sum_{n=0}^{\infty}\left\|f_{n}\right\|_{X}\left\|g_{n}\right\|_{Y} .
$$

The norm in $X \otimes Y$ is based on Schatten's definition of greatest crossnorm.

Theorem 2.2. If $X$ and $Y$ are $\mathcal{S}$-admissible space then $X \otimes Y$ is an $\mathcal{S}$ admissible Banach space.

Proof. Let us first show that the functional $\|\cdot\|_{X \otimes Y}$ is actually a norm.

Only the implication $\|h\|=0 \Longrightarrow h=0$ requires a proof. Let $\|h\|_{X \otimes Y}=0$. Let $\varepsilon>0$. Then $h=\sum_{n=0}^{\infty} f_{n} * g_{n}$, where $\sum_{n=0}^{\infty}\left\|f_{n}\right\|_{X}\left\|g_{n}\right\|_{Y}<\varepsilon$. Since $X$ and $Y$ are continuously embedded in $\mathcal{S}$, we have $\left|\hat{f}_{n}(j)\right| \leq C_{j}\left\|f_{n}\right\|_{X}$ and $\left|\hat{g}_{n}(j)\right| \leq D_{j}\left\|g_{n}\right\|_{Y}$, where $C_{j}$ and $D_{j}$ are constant depending only on $j$. Hence

$$
|\hat{h}(j)|=\left|\sum_{n=0}^{\infty} \hat{f}_{n}(j) \hat{g}_{n}(j)\right| \leq \sum_{n=0}^{\infty} C_{j} D_{j}\left\|f_{n}\right\|_{X}\left\|g_{n}\right\|_{Y} \leq C_{j} D_{j} \varepsilon .
$$

Thus $\hat{h}(j)=0$ because $\varepsilon$ was arbitrary.

Incidentally, this shows also that $X \otimes Y \subset \mathcal{S}$ with continuity. The fact that $\mathcal{P} \subset X \otimes Y$ is immediate. It remains to show that the space $X \otimes Y$ is complete.

Let $h_{n} \in X \otimes Y(n \geq 0)$ be such that $\sum_{n=0}^{\infty}\left\|h_{n}\right\|_{X \otimes Y}<\infty$. We have $h_{n}=\sum_{k=0}^{\infty} f_{k, n} * g_{k, n}$, where $\sum_{k=0}^{\infty}\left\|f_{k, n}\right\|_{X}\left\|g_{k, n}\right\|_{Y} \leq 2\left\|h_{n}\right\|$. It is easily verified that $h:=\sum_{n=0}^{\infty} h_{n}$ converges in $\mathcal{S}$ and therefore $h \in X \otimes Y$. It remains to prove that

$$
\left\|\sum_{n=m}^{\infty} h_{n}\right\|_{X \otimes Y} \rightarrow 0, \quad m \rightarrow \infty
$$

But this follows from

$$
\left\|\sum_{n=m}^{\infty} h_{n}\right\|_{X \otimes Y} \leq \sum_{k=0}^{\infty} \sum_{n=m}^{\infty}\left\|f_{k, n}\right\|_{X}\left\|g_{k, n}\right\|_{Y} \leq \sum_{n=m}^{\infty} 2\left\|h_{n}\right\|,
$$

concluding the proof. 
Proposition 2.1. If $\mathcal{P}$ is dense in $X$ or $Y$, then $\mathcal{P}$ is a dense subset of $X \otimes Y$. In particular $\left(X_{\mathcal{P}} \otimes Y\right)_{\mathcal{P}}=X_{\mathcal{P}} \otimes Y$.

Proof. By symmetry of the definition, let assume that $\mathcal{P}$ is dense in $X$. Let $h \in X \otimes Y$, and $\varepsilon>0$. Then, by the definition, there are a positive integer $n$ and $f_{k} \in X, g_{k} \in Y(0 \leq k \leq n)$ such that

$$
\left\|h-\sum_{k=0}^{n} f_{k} * g_{k}\right\|_{X \otimes Y}<\varepsilon / 2 .
$$

Choose polynomials $P_{k}$ so that $\left\|f_{k}-P_{k}\right\|_{X}<\varepsilon /(2 n)\left\|g_{k}\right\|_{Y}$. Then we have

$$
\begin{aligned}
\left\|h-\sum_{k=0}^{n} P_{k} * g_{k}\right\|_{X \otimes Y} & \leq\left\|h-\sum_{k=0}^{n} f_{k} * g_{k}\right\|_{X \otimes Y}+\left\|\sum_{k=0}^{n}\left(f_{k}-P_{k}\right) * g_{k}\right\|_{X \otimes Y} \\
& \leq \varepsilon / 2+\sum_{k=0}^{n}\left\|f_{k}-P_{k}\right\|_{X}\left\|g_{k}\right\|_{Y} \leq \varepsilon
\end{aligned}
$$

This concludes the proof because $\sum_{k=0}^{n} P_{k} * g_{k}$ is a polynomial.

The following fact can help in determining $X \otimes Y$ in simple situations. Recall that a quasinorm on a (complex) vector space $A$ is a functional $\|\cdot\|$ on $A$ satisfying the following conditions:

(i) $\|f\| \geq 0 ;\|f\|=0$ iff $f=0$.

(ii) $\|t f\|=|t|\|f\|$, for all $t \in \mathbb{C}, f \in A$.

(iii) $\|f+g\| \leq K(\|f\|+\|g\|)$ for all $f, g \in A$, where $K \geq 1$ is a constant.

The couple $(A,\|\cdot\|)$ is called a quasi-normed space. A complete quasinormed space is called a quasi-Banach space. "Complete" means that if $\left\{f_{k}\right\} \subset A$ is a sequence such that $\lim _{m, k}\left\|f_{m}-f_{k}\right\|=0$, then there is $f \in A$ such that $\lim _{k}\left\|f_{k}-f\right\|=0$. If $A^{\prime}$, the space of all bounded linear functionals on $A$, separates points in $A$, then there is the smallest Banach space, $[A]$, such that $A^{\prime}=[A]^{\prime}$. More precisely, let

$$
\|f\|_{1}=\sup \left\{|\Lambda f|: \Lambda \in A^{\prime},\|\Lambda\| \leq 1\right\} .
$$

Then $\|\cdot\|_{1}$ is a norm on $A$, and we define $[A]$ to be the completion of $\left(A,\|\cdot\|_{1}\right)$.

If $A \subset \mathcal{S}$ with continuous inclusion, then the dual $A^{\prime}$ separates points in $A$ because $f \mapsto \hat{f}(j)$, for each $j$, is in $A^{\prime}$. Then we can realize $[A]$ as the subset of $\mathcal{S}$ consisting of those $f$ that can be represented in the form

$$
f=\sum_{n=1}^{\infty} f_{n} \quad \text { with } \sum_{n=1}^{\infty}\left\|f_{n}\right\|_{A}<\infty .
$$


Moreover we have

$$
\|f\|_{[A]}=\inf \sum_{n=1}^{\infty}\left\|f_{n}\right\|_{A},
$$

where the infimum is taken over all representations of the form $(\ddagger)$. It follows from the condition $\sum_{n}\left\|f_{n}\right\|_{A}<\infty$ that the series $\sum_{n} f_{n}$ converges in $\mathcal{S}$.

Proposition 2.2. Let $X$ and $Y$ be $\mathcal{S}$-admissible Banach spaces.

(i) If there exists a Banach space $Z$ such that

$$
X * Y=\{f * g: f \in X, g \in Y\} \subset Z,
$$

then $X \otimes Y \subset Z$.

(ii) If $X * Y=A$ is a quasi-Banach space then $X \otimes Y=[A]$.

Proof. (i) An application of the closed graph theorem to the operators $f \mapsto f * g$ shows that

$$
\sup _{\|f\|_{X} \leq 1}\|f * g\|_{Z}<\infty
$$

Hence, by the Banach-Steinhauss theorem,

$$
\sup _{\|f\|_{X} \leq 1,\|g\|_{Y} \leq 1}\|f * g\|_{Z}<\infty .
$$

Now, assuming that $X * Y \subset Z$, let

$$
\sum_{j=1}^{\infty}\left\|f_{n}\right\|_{X}\left\|g_{n}\right\|_{Y}<\infty
$$

where $f_{n} \in X, g_{n} \in Y$. From this and $(\dagger)$ we obtain

$$
\sum_{n=1}^{\infty}\left\|f_{n} * g_{n}\right\|_{Z}<\infty
$$

whence $\sum_{n} f_{n} * g_{n}$ converges in $Z$. The result follows.

(ii) Let $X * Y=A$. Since $A \subset[A]$, we have $X \otimes Y \subset[A]$, by (i).

In the other direction, let $f \in[A]$. Choose $\left\{f_{n}\right\}_{1}^{\infty} \subset X * Y=A$ so that

$$
f=\sum_{n=0}^{\infty} f_{n} \quad \text { and }\|f\|_{[A]} \leq 2 \sum_{n=1}^{\infty}\left\|f_{n}\right\|_{A} .
$$

Choose $g_{n} \in X$ and $h_{n} \in Y$ so that $f_{n}=g_{n} * h_{n}$. Then, as above, $\left\|g_{n} * h_{n}\right\|_{A}$ $\leq C\left\|g_{n}\right\|_{X}\left\|h_{n}\right\|_{Y}$, where $C$ is independent of $n$. The result now follows. 
Corollary 2.1. Let $1 \leq p, q \leq \infty$ and $p * q=\max \left\{\frac{p q}{p+q}, 1\right\}$ where $\frac{p q}{p+q}=\infty$ if $p=\infty$ or $q=\infty$. Then $\ell^{p} \otimes \ell^{q}=\ell^{p * q}$.

Proof. It is easily seen that, for $p, q>0$,

$$
\ell^{p} * \ell^{q}=\ell^{s}, \quad \text { where } \frac{1}{s}=\frac{1}{p}+\frac{1}{q} .
$$

The result now follows from Proposition 2.2.

Here there is a basic formula connecting tensors and multipliers.

Theorem 2.3. Let $X, Y, Z$ be $\mathcal{S}$-admissible Banach spaces. Then

$$
(X \otimes Y, Z)=(X,(Y, Z)) .
$$

Proof. Let $\lambda \in(X \otimes Y, Z)$. We have to prove that $\lambda * f \in(Y, Z)$, for all $f \in X$, i.e., that $\lambda * f * g \in Z$, for all $f \in X, g \in Y$. But, since $f * g \in X \otimes Y$, the hypothesis $\lambda \in(X \otimes Y, Z)$ implies $\lambda *(f * g) \in Z$. Hence we have proved that $(X \otimes Y, Z) \subset(X,(Y, Z))$.

In the other direction, assume that $\lambda \in(X,(Y, Z))$, and let $h \in X \otimes Y$. Then

$$
h=\sum_{n=1}^{\infty} f_{n} * g_{n}, \quad f_{n} \in X, g_{n} \in Y,
$$

and

$$
\sum_{n=1}^{\infty}\left\|f_{n}\right\|_{X}\left\|g_{n}\right\|_{Y} \leq 2\|h\|_{X \otimes Y}
$$

Hence $\lambda * h=\sum_{n=1}^{\infty} \lambda * f_{n} * g_{n}$ (convergence in $\mathcal{S}$ ). Since $\lambda * f_{n} \in(Y, Z)$, we have $\lambda * f_{n} * g_{n} \in Z$, whence

$$
\left\|\sum_{n=1}^{\infty} \lambda * f_{n} * g_{n}\right\|_{Z} \leq\left\|\lambda * f_{n}\right\|_{(Y, Z)}\left\|g_{n}\right\|_{Y} \leq\|\lambda\|_{(X,(Y, Z))}\left\|f_{n}\right\|_{X}\left\|g_{n}\right\|_{Y}<\infty .
$$

Since $Z$ is complete we have that

$$
\lambda * \sum_{n=1}^{\infty} f_{n} * g_{n}=\sum_{n=1}^{\infty} \lambda * f_{n} * g_{n} \in Z,
$$

i.e., $\lambda \in(X \otimes Y, Z)$. This completes the proof of the theorem.

Corollary 2.2. Let $X$ and $Y$ be $\mathcal{S}$-admissible Banach spaces. Then

$$
(X \otimes Y)^{K}=\left(X, Y^{K}\right),(X \otimes Y)^{*}=\left(X, Y^{*}\right) .
$$




\section{3. $\mathcal{H}$-admissible Banach spaces}

Definition 3.1. A Banach space $X \subset \mathcal{S}$ is said to be $\mathcal{H}$-admissible if

(i) $X \subset \mathcal{H}(\mathbb{D})$ with continuous inclusion, and

(ii) $\mathcal{H}\left(\mathbb{D}_{R}\right) \subset X$ for each $R>1$ and $\left.f \mapsto f\right|_{\mathbb{D}}$ is continuous from $\mathcal{H}\left(\mathbb{D}_{R}\right)$ to $X$.

Proposition 3.1. Let $X$ be $\mathcal{H}$-admissible. Then

(i) $C_{X}(z)=\sum_{n=0}^{\infty} e_{n} z^{n} \in \mathcal{H}(\mathbb{D}, X)$.

(ii) $C_{X^{\prime}}(z)=\sum_{n=0}^{\infty} \gamma_{n} z^{n} \in \mathcal{H}\left(\mathbb{D}, X^{\prime}\right)$.

(iii) The mapping $f \rightarrow F$ where $F(w)=f_{w}$ defines a continuous inclusion $X \subset \mathcal{H}\left(\mathbb{D}, X_{\mathcal{P}}\right)$.

Proof. (i) Observe first if $X$ is $\mathcal{H}$-admissible then for any $0<r<1$ there is a constant $A_{r}<\infty$, depending only on $r$, such that

$$
M_{\infty}(r, f) \leq A_{r}\|f\|_{X}, \quad f \in X .
$$

In particular, $r^{n} \leq A_{r}\left\|e_{n}\right\|$ for all $n \in \mathbb{N}$. On the other hand, for each $R>1$ and $f \in \mathcal{H}\left(\mathbb{D}_{R}\right)$ then $f \in X$ and there exists $C_{R}>0$ such that

$$
\|f\|_{X} \leq C_{R} \sup _{|z|<R}|f(z)|,
$$

equivalently if $f \in \mathcal{H}(\mathbb{D})$ then $f_{r} \in X$, for every $r \in(0,1)$, and there holds the inequality

$$
\left\|f_{r}\right\|_{X} \leq B_{r}\|f\|_{\infty} \quad(0<r<1) .
$$

In particular, $r^{-n}\left\|e_{n}\right\|_{X} \leq B_{r}$ for all $n \in \mathbb{N}$.

From these estimates one easily deduces that

$$
\lim _{n \rightarrow \infty} \sqrt[n]{\left\|e_{n}\right\|_{X}}=1
$$

Therefore (i) follows.

(ii) On the other hand

$$
\left\|\gamma_{n}\right\|_{X^{\prime}}=\sup _{\|f\|_{X} \leq 1}|\hat{f}(n)| \leq r^{-n} A_{r}
$$

and $1 \leq\left\|\gamma_{n}\right\|_{X^{\prime}}\left\|e_{n}\right\|_{X}$. This gives

$$
\lim _{n \rightarrow \infty} \sqrt[n]{\left\|\gamma_{n}\right\|_{X^{\prime}}}=1,
$$

which implies (ii). 
(iii) It follows from (i) that if $f \in X$ then

$$
f_{w}=\sum_{n=0}^{\infty} \gamma_{n}(f) e_{n} w^{n}
$$

is absolutely convergent in $X$. Hence $f_{w} \in X_{\mathcal{P}}$ for any $w \in \mathbb{D}$ and $w \rightarrow f_{w}$ is an $X_{\mathcal{P}}$-valued analytic function on the unit disk $\mathbb{D}$.

Proposition 3.2. Let $X$ is $\mathcal{H}$-admissible and, for $0<r<1$, write

$$
M_{X}(r, f)=\sup _{|w|=r}\left\|f_{w}\right\|_{X}
$$

Then

(i) $M_{X}(r, f)$ is increasing.

(ii) $M_{\infty}(r, f) \leq A_{X}(r)\|f\|_{X}, f \in X$, where $A_{X}(r)=\left\|\left(C_{X^{\prime}}\right)_{r}\right\|_{C\left(\mathbb{T}, X^{\prime}\right)}$.

(iii) $M_{X}(r, f) \leq B_{X}(r)\|f\|_{\infty}, f \in A(\mathbb{D})$, where $B_{X}(r)=\left\|\left(C_{X}\right)_{r}\right\|_{L^{1}(\mathbb{T}, X)}$.

Proof. (i) Since $F(w)=f_{w} \in \mathcal{H}(\mathbb{D}, X)$ then $w \rightarrow\|F(w)\|_{X}$ is subharmonic. Therefore $M_{X}(r, f)=\sup _{|w|=r}\left\|f_{w}\right\|_{X}$ is increasing in $r$.

(ii) Note that $C_{X^{\prime}}(z)=\sum_{n=0}^{\infty} \gamma_{n} z^{n} \in \mathcal{H}\left(\mathbb{D}, X^{\prime}\right)$ and, for each $0<r<1$, the series $\left(C_{X^{\prime}}\right)_{r}(z)=\sum_{n=0}^{\infty} \gamma_{n} z^{n} r^{n}$ is absolutely convergent in $C\left(\mathbb{T}, X^{\prime}\right)$. Hence

$$
f_{r}(z)=\sum_{n=0}^{\infty} \gamma_{n}(f) r^{n} e_{n}(z)=\left(C_{X^{\prime}}\right)_{r}(z)(f),
$$

which implies that $M_{\infty}(r, f) \leq A_{X}(r)\|f\|_{X}$.

(iii) We write, for $f \in A(\mathbb{D})$,

$$
f_{w}=\int_{0}^{2 \pi} f\left(e^{-i \theta}\right) C_{w e^{i \theta}} \frac{d \theta}{2 \pi} .
$$

Now, for $|w|=r$, applying Minkowski's inequality

$$
\left\|f_{w}\right\|_{X} \leq \int_{0}^{2 \pi}\left|f\left(e^{-i \theta}\right)\right|\left\|C_{w e^{i \theta}}\right\|_{X} \frac{d \theta}{2 \pi} \leq\|f\|_{\infty} \int_{0}^{2 \pi}\left\|\left(C_{X}\right)_{r}\left(e^{i \theta}\right)\right\|_{X} \frac{d \theta}{2 \pi} .
$$

This gives the result.

Given $v: \mathbb{D} \rightarrow[0, \infty)$ a continuous weight, let $H_{v}^{\infty}$ denote the space of $f \in \mathcal{H}(\mathbb{D})$ such that $\sup _{z \in \mathbb{D}} v(z)|f(z)|<\infty$. Hence (ii) in Proposition 3.2 shows the following fact.

Corollary 3.1. Let $X$ be $\mathcal{H}$-admissible and define $v_{1}^{-1}(z)=A_{X}(|z|)=$ $\left\|\left(C_{X^{\prime}}\right)_{|z|}\right\|_{C\left(\mathbb{T}, X^{\prime}\right)}$. Then $X \subset H_{v_{1}}^{\infty}$ with continuous inclusion. 
Let us now show that also taking multipliers and tensors preserve $\mathcal{H}$ admissiblity.

Theorem 3.1. Let $X$ and $Y$ be $\mathcal{H}$-admissible. Then $(X, Y)$ and $X \otimes Y$ are $\mathcal{H}$-admissible Banach spaces.

Proof. Let us take $\lambda \in(X, Y)$ and observe that, using Proposition 3.2,

$$
M_{\infty}(r, \lambda) \leq A_{Y}(r)\left\|\lambda * C_{r}\right\|_{Y} \leq A_{Y}(r)\|\lambda\|_{(X, Y)}\left\|C_{r}\right\|_{X} .
$$

This gives that $(X, Y) \subset \mathcal{H}(\mathbb{D})$ with continuity.

Also note that if $\lambda \in \mathcal{H}(\mathbb{D})$ then

$$
\begin{aligned}
\left\|\lambda_{r^{2}}\right\|_{(X, Y)} & =\sup _{\|f\|_{X} \leq 1}\left\|\left(\lambda * f_{r}\right)_{r}\right\|_{Y} \leq B_{Y}(r) \sup _{\|f\|_{X} \leq 1} M_{\infty}(r, \lambda * f) \\
& \leq B_{Y}(r)\|\lambda\|_{\infty} \sup _{\|f\|_{X} \leq 1} M_{\infty}(r, f) \leq B_{Y}(r) A_{X}(r)\|\lambda\|_{\infty} .
\end{aligned}
$$

This is equivalent to $\mathcal{H}\left(\mathbb{D}_{R}\right) \subset(X, Y)$ for any $R>1$.

To show that $X \otimes Y$ is $\mathcal{H}$-admissible Let $h=\sum_{n=0}^{\infty} f_{n} * g_{n}$ where the series converges in $\mathcal{S}$ and $\sum_{n=0}^{\infty}\left\|f_{n}\right\|_{X}\left\|g_{n}\right\|_{Y}<\infty$. Observe that for each $0<r<1$

$$
h_{r^{2}}=\sum_{n=0}^{\infty}\left(f_{n}\right)_{r} *\left(g_{n}\right)_{r}
$$

Hence

$$
M_{\infty}\left(r^{2}, h\right) \leq \sum_{n=0}^{\infty} M_{\infty}\left(r, f_{n}\right) M_{\infty}\left(r, g_{n}\right) \leq A_{X}(r) A_{Y}(r) \sum_{n=0}^{\infty}\left\|f_{n}\right\|_{X}\left\|g_{n}\right\|_{Y} .
$$

Hence, taking the infimum over all representations,

$$
M_{\infty}\left(r^{2}, h\right) \leq A_{X}(r) A_{Y}(r)\|h\|_{X \otimes Y} .
$$

This shows that $X \otimes Y \subset \mathcal{H}(\mathbb{D})$ with continuity.

Let us now take $h \in \mathcal{H}\left(\mathbb{D}_{R}\right)$ and fix $1<S<R$. Hence $\sum_{n=0}^{\infty}|\hat{h}(n)| S^{n}<\infty$. Using that $\lim _{n \rightarrow \infty} \sqrt[n]{\left\|e_{n}\right\|_{X}\left\|e_{n}\right\|_{Y}}=1$, we can write $h=\sum_{n=0}^{\infty} \hat{h}(n) e_{n} * e_{n}$, with convergence in $\mathcal{H}(\mathbb{D})$ and

$$
\sum_{n=0}^{\infty}\left\|\hat{h}(n) e_{n}\right\|_{X}\left\|e_{n}\right\|_{Y} \leq K \sum_{n=0}^{\infty} S^{-n}\left\|e_{n}\right\|_{X}\left\|e_{n}\right\|_{Y}<\infty .
$$

Definition 3.2. If $X$ is an $\mathcal{H}$-admissible Banach space we define $\tilde{X}$ as the space of functions in $\mathcal{H}(\mathbb{D})$ such that $w \rightarrow f_{w} \in H^{\infty}(\mathbb{D}, X)$. We write

$$
\|f\|_{\tilde{X}}=\sup _{0<r<1} M_{X}(r, f) .
$$

For instance $\widetilde{H^{p}}=H^{p}$ or $\widetilde{A(\mathbb{D})}=H^{\infty}$. 
Let us collect some properties of $\tilde{X}$ in the next proposition.

Proposition 3.3. Let $X \subset \mathcal{H}(\mathbb{D})$ be $\mathcal{H}$-admissible. Then

(i) $\tilde{X}$ is $\mathcal{H}$-admissible.

(ii) $\tilde{X}_{\mathcal{P}} \subset X_{\mathcal{P}}$ and $\tilde{X}=\widetilde{\left(X_{\mathcal{P}}\right)}=\tilde{\tilde{X}}$.

(iii) $X^{\#} \subset X^{*} \subset\left(X_{\mathcal{P}}\right)^{\#} \subset(\tilde{X})^{*}$ with continuous inclusions. In particular $\left(X_{\mathcal{P}}\right)^{*}=\left(X_{\mathcal{P}}\right)^{\#}$.

Proof. (i) The fact that $\|\cdot\|_{\tilde{X}}$ is a norm and complete is standard. Due to (i) in Proposition 3.2 one has that for $0<r<1$

$$
M_{\tilde{X}}(r, f)=\left\|f_{r}\right\|_{\tilde{X}}=M_{X}(r, f) .
$$

From this one easily shows that $\tilde{X}$ is also $\mathcal{H}$-admissible.

(ii) Note that

$$
\left\|f_{r}\right\|_{\tilde{X}}=M_{X_{\mathcal{P}}}(r, f)=M_{\tilde{X}}(r, f),
$$

which gives that $\tilde{X}=\widetilde{X_{\mathcal{P}}}$. On the other hand if $f \in \mathcal{P}$ then

$$
\|f\|_{X}=\lim _{r \rightarrow 1}\left\|f_{r}\right\|_{X} \leq \sup _{0<r<1} M_{X}(r, f)=\|f\|_{\tilde{X}} .
$$

(iii) The first inclusion is immediate. For the second one note that $\left(H^{\infty}\right)_{\mathcal{P}}=A(\mathbb{D})$ and that $(X, Y) \subset\left(X_{\mathcal{P}}, Y_{\mathcal{P}}\right)$.

Let $g \in\left(X_{\mathcal{P}}\right)^{\#}$. Since $f_{r} \in X_{\mathcal{P}}$ one has

$$
\left\|(g * f)_{r}\right\|_{A(\mathbb{D})} \leq C\left\|f_{r}\right\|_{X} \leq C\|f\|_{\tilde{X}}
$$

This shows that $g \in(\tilde{X})^{*}$.

Let us now present some useful lemmas to be used in the sequel.

Lemma 3.1. Let $X \subset \mathcal{H}(\mathbb{D})$ be an $\mathcal{H}$-admissible Banach space. If $f, g \in$ $\mathcal{H}(\mathbb{D})$ then

$$
M_{X}(r s, f * g) \leq M_{1}(r, f) M_{X}(s, g),
$$

Proof. Let $0 \leq r, s<1,|w|=r$ and $\left|w^{\prime}\right|=s$

$$
(f * g)_{w w^{\prime}}=\sum_{n=0}^{\infty} \gamma_{n}\left(f_{w}\right) \gamma_{n}\left(g_{w}\right) e_{n}
$$

where the series is absolutely convergent in $X$. Hence one concludes

$$
(f * g)_{w w^{\prime}}=\int_{0}^{2 \pi} f\left(w e^{-i \theta}\right) g_{w^{\prime} e^{i \theta}} \frac{d \theta}{2 \pi}
$$


where the integral is understood in the vector valued sense. Using Minkowski's inequality

$$
\left\|(f * g)_{w w^{\prime}}\right\|_{X} \leq \int_{0}^{2 \pi}\left|f\left(w e^{-i \theta}\right)\right|\left\|g_{w^{\prime} e^{i \theta}}\right\|_{X} \frac{d \theta}{2 \pi} \leq M_{1}(r, f) M_{X}(s, g) .
$$

This implies the result.

Lemma 3.2. Let $X \subset \mathcal{H}(\mathbb{D})$ be an $\mathcal{H}$-admissible Banach space and $f \in$ $\mathcal{H}(\mathbb{D})$. Then

$$
\begin{aligned}
M_{X}(r s, D f) & \leq \frac{1}{1-r^{2}} M_{X}(s, f), \\
M_{X}(r, f) d r & \leq \int_{0}^{1} M_{X}(r s, D f) d s,
\end{aligned}
$$

where $\operatorname{Df}(z)=\sum_{n=1}^{\infty}(n+1) \hat{f}(n) z^{n}$.

Proof. Recall that $D e_{n}=(n+1) e_{n}$ and $D f=K * f$ where $K(z)=\frac{1}{(1-z)^{2}}$. Use Lemma 3.1 to obtain (3.1).

To see (3.2) simply use that, for each $0 \leq r<1$ and $|\xi|=1$, one has

$$
r f_{r \xi}=\int_{0}^{r}(D f)_{s \xi} d s
$$

as $X$-valued function. Hence, by Minkowski's inequality,

$$
r M_{X}(r, f) d r \leq \int_{0}^{r} M_{X}(s, D f) d s=r \int_{0}^{1} M_{X}(r s, D f) d s .
$$

Definition 3.3. If $X$ is an $\mathcal{H}$-admissible Banach space and $1 \leq q<\infty$ we write $\mathfrak{B}^{X, q}$ for the spaces of holomorphic functions such that

$$
\|f\|_{\mathfrak{B}^{X, q}}=\left(\int_{0}^{1}\left(1-r^{2}\right)^{q-1} M_{X}^{q}(r, D f) r d r\right)^{1 / q}<\infty .
$$

The case $q=\infty$ corresponds to

$$
\|f\|_{\mathfrak{B}^{X, \infty}}=\sup _{0<r<1}\left(1-r^{2}\right) M_{X}(r, D f) .
$$

Clearly $\mathfrak{B}^{H^{p}, q}$ coincides with $\mathfrak{B}^{p, q}, 1 \leq p, q \leq \infty$, consisting of those $f \in \mathcal{H}(\mathbb{D})$ for which

$$
\|f\|_{\mathfrak{B}^{p, q}}:=\left(|f(0)|^{q}+\int_{0}^{1} M_{p}^{q}\left(r, f^{\prime}\right)(1-r)^{q-1} r d r\right)^{1 / q}<\infty .
$$


(These spaces are called in [14] Hardy-Bloch spaces.) In the case $q=\infty$, this should be interpreted as

$$
|f(0)|+\sup _{0<r<1} M_{p}\left(r, f^{\prime}\right)(1-r)<\infty .
$$

Clearly $\mathfrak{B}^{\infty, \infty}$ coincides with the Bloch space $\mathfrak{B}$.

It is easy to see that also $\mathfrak{B}^{\ell^{q}, q}=\ell^{q}$.

Definition 3.4. Let $0<p, q \leq \infty$. The space $\ell(p, q)$ introduced by Kellogg $([18])$, consists of complex sequences $\{\hat{a}(k)\}_{0}^{\infty}$ such that

$$
\left\{\left(\sum_{j \in I_{k}}|\hat{a}(j)|^{p}\right)^{1 / p}\right\}_{k=0}^{\infty} \in \ell^{q}
$$

where $I_{k}=\left\{j: 2^{k-1} \leq j<2^{k}\right\}$, for $k \geq 1$, and $I_{0}=\{0\}$. The quasinorm in $\ell(p, q)$ is given by

$$
\|\{\hat{a}(j)\}\|_{\ell(p, q)}=\left\|\left\{\left(\sum_{j \in I_{k}}|\hat{a}(j)|^{p}\right)^{1 / p}\right\}_{k=0}^{\infty}\right\|_{\ell^{q}} .
$$

It follows that $\ell(p, p)$ is identical with $\ell^{p}$. It is not difficult to show that, for $q<\infty$, the dual of $\ell(p, q)$ is (isometrically) isomorphic to $\ell\left(p^{\prime}, q^{\prime}\right)$, with the duality pairing given by

$$
(a, b) \mapsto \sum_{j=0}^{\infty} \hat{a}(j) \hat{b}(j)
$$

(the series being absolutely convergent), where $1 / p^{\prime}=1-1 / p$ for $p \in(1, \infty]$ and $p^{\prime}=\infty$ for $p \leq 1$. Hence, the norm in $\ell(p, q)$, where $1 \leq p \leq \infty$ and $1 \leq q<\infty$, can be given by means of the formula

$$
\|a\|_{\ell(p, q)}=\sup \left\{\left|\sum_{j=0}^{\infty} \hat{a}(j) \hat{b}(j)\right|:\|b\|_{\ell\left(p^{\prime}, q^{\prime}\right)} \leq 1\right\} .
$$

This can be used to derive the following formula for the Banach envelope of $\ell(p, q)$ :

$$
[\ell(p, q)]= \begin{cases}\ell^{1}, & \text { if } p, q \leq 1, \\ \ell(p, 1), & \text { if } 1<p \leq \infty, q<1, \\ \ell(1, q), & \text { if } p<1,1<q \leq \infty\end{cases}
$$


Given $0<u, v<\infty$ let us denote

$$
u \ominus v= \begin{cases}\frac{u v}{u-v}, & \text { if } v<u<\infty, \\ v, & \text { if } u=\infty \\ \infty & \text { if } u \leq v\end{cases}
$$

(The notation $u \ominus v$ was introduced in [7].) Kellogg proved the following extension of Hölder duality result.

Proposition 3.4. Let $1 \leq p_{1}, p_{2}, q_{1}, q_{2} \leq \infty$. Then

$$
\left(\ell\left(p_{1}, q_{1}\right), \ell\left(p_{2}, q_{2}\right)\right)=\ell\left(p_{1} \ominus p_{2}, q_{1} \ominus q_{2}\right)
$$

with equal norms.

It is not hard to generalize the formula (2.2) to the setting of the Kellogg spaces.

$$
\ell\left(p_{1}, q_{1}\right) * \ell\left(p_{2}, q_{2}\right)=\ell\left(s_{1}, s_{2}\right)
$$

where

$$
\frac{1}{s_{j}}=\frac{1}{p_{j}}+\frac{1}{q_{j}} .
$$

Then, using Proposition 2.2 and formula (3.3), one proves the following result.

Proposition 3.5. Let $1 \leq p_{j}, q_{j} \leq \infty$. Then

$$
\ell\left(p_{1}, q_{1}\right) \otimes \ell\left(p_{2}, q_{2}\right)=\ell\left(p_{1} * p_{2}, q_{1} * q_{2}\right) .
$$

Proposition 3.6. Let $1 \leq p, q \leq \infty$. Then $\mathfrak{B}^{\ell^{p}, q}=\ell(p, q)$.

Proof. The case $q=\infty$ follows from the observation that $f \in \ell(p, \infty)$ can be rewritten by the condition

$$
\sum_{n=0}^{\infty}|(n+1) \hat{f}(n)|^{p} r^{n p} \leq \frac{C}{(1-r)^{p}} .
$$

The case $q<\infty$ follows from the inequalities, for $p, \alpha>0$ and $a_{k} \geq 0$, (see [20] or also [4, Lemma 2.1])

$$
\begin{aligned}
A_{p, \alpha} \sum_{n=0}^{\infty} 2^{-n \alpha}\left(\sum_{k \in I_{n}} a_{k}\right)^{p} & \leq \int_{0}^{1}(1-r)^{p \alpha-1}\left(\sum_{k=0}^{\infty} a_{k} r^{k}\right)^{p} d r \\
& \leq B_{p, \alpha} \sum_{n=0}^{\infty} 2^{-n \alpha}\left(\sum_{k \in I_{n}} a_{k}\right)^{p}
\end{aligned}
$$




\section{Homogeneous spaces of analytic functions}

Definition 4.1. Let $X$ be an $\mathcal{H}$-admissible Banach space. It is said to be homogeneous if it satisfies:

(i) If $f \in X$ and $|\xi|=1$, then $f_{\xi} \in X$ and $\left\|f_{\xi}\right\|_{X}=\|f\|_{X}$.

(ii) If $f \in X$ and $0<r<1$ then $M_{X}(r, f) \leq K\|f\|_{X}$, where $K$ is a constant independent of $f$ and $r$.

Observe that for homogeneous spaces $C_{\xi} \in(X, X)$ with $\left\|C_{\xi}\right\|_{(X, X)}=1$ if $|\xi|=1$ and $C_{r} \in(X, X)$ with $\sup _{0<r<1}\left\|C_{r}\right\|_{(X, X)} \leq K$. Note also that in this case $\left\|f_{w}\right\|_{X}=\left\|f_{|w|}\right\|_{X}$ and $\left\|f_{r}\right\|=M_{X}(r, f)$ and $X \subset \tilde{X}$ with continuity.

We denote by $H^{\infty}(\mathbb{D}, X)$ the space of $X$-valued bounded analytic functions, and $A(\mathbb{D}, X)$ those with continuous extension to the boundary, i.e. the closure of $X$-valued polynomials.

Proposition 4.1. Let $X$ be an homogeneous Banach space.

(i) If $f \in X$ then $w \rightarrow f_{w} \in H^{\infty}\left(\mathbb{D}, X_{\mathcal{P}}\right)$.

(ii) If $f \in X_{\mathcal{P}}$ then $w \rightarrow f_{w} \in A\left(\mathbb{D}, X_{\mathcal{P}}\right)$.

Proof. (i) Note that the $\mathcal{H}$-admissibility guarantees that $F(w)=f_{w} \in$ $\mathcal{H}\left(\mathbb{D}, X_{\mathcal{P}}\right)$. For homogeneous spaces

$$
M_{X}(r, f)=\sup _{|\xi|=1}\left\|f_{r \xi}\right\|_{X}=\left\|F_{r}\right\|_{H^{\infty}(\mathbb{D}, X)} .
$$

Hence $F \in H^{\infty}(\mathbb{D}, X)$.

(ii) It is clear that if $f \in X_{\mathcal{P}}$ then $\lim _{r \rightarrow 1}\left\|f_{r}-f\right\|=0$. Now use that $\left\|F-F_{r}\right\|_{H^{\infty}(\mathbb{D}, X)}=\left\|f-f_{r}\right\|$ to conclude the result, because $F_{r} \in A(\mathbb{D}, X)$ for each $0<r<1$.

Proposition 4.2. Let $X$ and $Y$ be $\mathcal{H}$-admissible Banach spaces. Then

(i) $\tilde{X}$ is homogeneous.

(ii) If $Y$ is homogeneous then $(X, Y)$ is homogeneous.

(iii) If $X$ and $Y$ are homogeneous then $X \otimes Y$ is homogeneous.

Proof. The $\mathcal{H}$-admissibility of $(X, Y), X \otimes Y$ and $\tilde{X}$ was proved in Theorems 3.1 and 3.3 respectively.

(i) To show that $\tilde{X}$ is homogeneous use that $M_{X}(r, f)$ is increasing and the facts, for $|\xi|=1$ and $0<r, s<1$,

$$
M_{X}\left(r, f_{\xi}\right)=M_{X}(r, f) \text { and } \quad M_{X}\left(s, f_{r}\right)=M_{X}(s r, f) .
$$


(ii) Given $\lambda \in(X, Y)$ and $f \in X$ one has that

$$
\lambda_{w} * f=(\lambda * f)_{w}
$$

what trivially gives the result using the properties of $Y$.

(iii) Now given $h \in X \otimes Y$ with $h=\sum_{n=0}^{\infty} f_{n} * g_{n}$ with $\sum_{n=0}^{\infty}\left\|f_{n}\right\|\left\|g_{n}\right\|<\infty$ one has

$$
M_{X \otimes Y}\left(r^{2}, h\right) \leq \sum_{n=1}^{\infty} M_{X}\left(r, f_{n}\right) M_{Y}\left(r, g_{n}\right) \leq K^{2} \sum_{n=1}^{\infty}\left\|f_{n}\right\|_{X}\left\|g_{n}\right\|_{Y} .
$$

Therefore $M_{X \otimes Y}\left(r^{2}, h\right) \leq\|h\|_{X \otimes Y}$ for all $0<r<1$.

Taking into account that

$$
h_{\xi}=\sum_{n=0}^{\infty}\left(f_{n}\right)_{\xi} * g_{n}, \quad|\xi|=1
$$

one concludes that $\left\|h_{\xi}\right\|_{X \otimes Y} \leq\|h\|_{X \otimes Y}$ for $|\xi|=1$. Therefore $\left\|h_{\xi}\right\|_{X \otimes Y}=$ $\|h\|_{X \otimes Y}$.

Proposition 4.3. Let $X$ be $\mathcal{H}$-admissible and $1 \leq q \leq \infty$. Then

(i) $\mathfrak{B}^{X, q}$ is homogeneous.

(ii) $\left(\mathfrak{B}^{X, q}\right)_{\mathcal{P}}=\mathfrak{B}^{X, q}$ for $1 \leq q<\infty$.

(iii) $\left(\mathfrak{B}^{X, \infty}\right)_{\mathcal{P}}=\left\{f \in \mathcal{H}(\mathbb{D}): \lim _{r \rightarrow 1}\left(1-r^{2}\right) M_{X}(r, D f)=0\right\}$.

(iv) $\mathfrak{B}^{X, 1} \subset X_{\mathcal{P}}$ and $\tilde{X} \subset \mathfrak{B}^{X, \infty}$.

Proof. (i) The facts that $\|\cdot\|_{\mathfrak{B}^{X, q}}$ is a norm and the completeness follow from standard arguments which are left to the reader. The $\mathcal{H}$-admissibility and homogeneity follow from the fact $\left\|f_{s}\right\|_{\mathfrak{B}^{X, q}}=M_{\mathfrak{B}^{X, q}}(s, f)$ and Lemmas 3.1 and 3.2.

(ii) Note that $\lim _{r \rightarrow 1} M_{X}\left(s, f_{r}-f\right)=0$ for each $0<s<1$. Hence, using the Lebesgue dominated convergence theorem, one sees that, for $q<\infty$, if $f \in \mathfrak{B}^{X, q}$ then $\left\|f_{r}-f\right\|_{\mathfrak{B}^{X, q}} \rightarrow 0$ as $r \rightarrow 1$. Since $f_{r} \in\left(\mathfrak{B}^{X, q}\right)_{\mathcal{P}}$ the result follows.

(iii) Since any polynomial $f \in \mathcal{P}$ satisfies that $\lim _{r \rightarrow 1}\left(1-r^{2}\right) M_{X}(r, D f)$ $=0$ then $\left(\mathfrak{B}^{X, \infty}\right)_{\mathcal{P}} \subset\left\{f \in \mathcal{H}(\mathbb{D}): \lim _{r \rightarrow 1}\left(1-r^{2}\right) M_{X}(r, D f)=0\right\}$. Let $f \in \mathcal{H}(\mathbb{D})$ such that $\lim _{r \rightarrow 1}\left(1-r^{2}\right) M_{X}(r, D f)=0$. For each $\varepsilon>0$ there exists $r_{0}<1$ such that

$$
\left(1-s^{2}\right) \sup _{r>s} M_{X}(r, D f)<\varepsilon, r_{0} \leq s<1
$$


Now observe that

$$
\begin{gathered}
\left\|f-f_{s}\right\|_{\mathfrak{B}^{X, \infty}} \leq M_{X}\left(r_{0}, D\left(f_{s}-f\right)\right)+2\left(1-s^{2}\right) \sup _{r>r_{0}} M_{X}(r, D f) \\
\leq M_{X}\left(r_{0}, D\left(f_{s}-f\right)\right)+\varepsilon .
\end{gathered}
$$

Therefore $f_{s} \in\left(\mathfrak{B}^{X, \infty}\right)_{\mathcal{P}}$ approaches $f$.

(iv) It follows from Lemma 3.2 and (ii).

Proposition 4.4. Let $X$ and $Y$ be homogeneous Banach spaces. Then

$$
\left(\mathfrak{B}^{X, 1}, Y\right)=\mathfrak{B}^{(X, Y), \infty} .
$$

Proof. Let $f$ be a polynomial and $g \in \mathfrak{B}^{(X, Y), \infty}$. Observe that

$$
\begin{aligned}
f * g(z) & =\frac{1}{2} \int_{0}^{1}\left(1-r^{2}\right) r^{2 n+1} \sum_{n=0}^{\infty}(n+1) n \hat{f}(n) \hat{g}(n) z^{n} \\
& =\frac{1}{2} \int_{0}^{1}\left(1-r^{2}\right)(D f)_{r} *\left((D g)_{r}-g_{r}\right)(z) r d r .
\end{aligned}
$$

Using that $M_{(X, Y)}(r, g) \leq M_{(X, Y)}(r, D g)$ (see (3.2)) one concludes that

$$
\begin{aligned}
\|f * g\|_{Y} & \leq \int_{0}^{1}\left(1-r^{2}\right)\left\|(D f)_{r} *\left((D g)_{r}-g_{r}\right)\right\|_{Y} r d r \\
& \leq \int_{0}^{1}\left(1-r^{2}\right) M_{(X, Y)}(r,(D g)-g) M_{X}(r, D f) r d r \\
& \leq 2 \int_{0}^{1} M_{X}(r,(D f))\left(1-r^{2}\right) M_{(X, Y)}(r, D g) r d r \\
& \leq 2\|f\|_{\mathfrak{B}^{X, 1}}\|g\|_{\mathfrak{B}^{(X, Y), \infty}} .
\end{aligned}
$$

Using that polynomials are dense in $\mathfrak{B}^{X, 1}$ one easily concludes that $\mathfrak{B}^{(X, Y), \infty}$ $\subset\left(\mathfrak{B}^{X, 1}, Y\right)$.

Let $f \in\left(\mathfrak{B}^{X, 1}, Y\right)$. Then

$$
\begin{aligned}
M_{(X, Y)}(r, D f) & =\sup \left\{\left\|D f * g_{r}\right\|_{Y}:\|g\|_{X} \leq 1\right\} \\
& =\sup \left\{\left\|f * D g_{r}\right\|_{Y}:\|g\|_{X} \leq 1\right\} \\
& \leq\|f\|_{\left(\mathfrak{B}^{X, 1}, Y\right)} \sup \left\{\left\|D g_{r}\right\|_{\mathfrak{B}^{X, 1}}:\|g\|_{X} \leq 1\right\} \\
& \leq\|f\|_{\left(\mathfrak{B}^{X, 1}, Y\right)} \sup \left\{\int_{0}^{1} M_{X}\left(s, D^{2} g_{r}\right) d s:\|g\|_{X} \leq 1\right\} .
\end{aligned}
$$

Observe now that

$$
\begin{aligned}
\int_{0}^{1} M_{X}\left(s, D^{2} g_{r}\right) s d s & =\int_{0}^{1} M_{X}\left(s r, D^{2} g\right) s d s \leq \int_{0}^{1} \frac{M_{X}(\sqrt{s r}, D g)}{1-s r} d s \\
& \leq A \int_{0}^{1} \frac{\|g\|_{X}}{(1-s r)^{2}} d s \leq A^{\prime \prime} \frac{\|g\|_{X}}{\left(1-r^{2}\right)}
\end{aligned}
$$

This estimate concludes the proof. 
Corollary 4.1. If $X$ is homogeneous then

$$
\left(\mathfrak{B}^{X, 1}\right)^{\#}=\left(\mathfrak{B}^{X, 1}\right)^{*}=\left(\mathfrak{B}^{X, 1}\right)^{\prime}=\mathfrak{B}^{X^{*}, \infty} \text { and }\left(\mathfrak{B}^{X, 1}\right)^{K}=\mathfrak{B}^{X^{K}, \infty} .
$$

Let us give some information on the dual of homogeneous Banach spaces.

Recall that we use the notation $A^{\#}=(X, A(\mathbb{D}))$. Hence, in particular $X^{\#} \subset X^{\prime}$ by means of $f \rightarrow \lambda * f(1)$ for $\lambda \in X^{\#}$.

Therefore we have the following chain of continuous inclusions between $\mathcal{H}$-admissible Banach spaces:

$$
X^{K} \subseteq X^{\#} \subseteq X^{\prime}
$$

Proposition 4.5. Let $X$ be an homogeneous Banach space. Then $X^{\#} \subset$ $\left(X_{\mathcal{P}}\right)^{\prime} \subset\left(X_{\mathcal{P}}\right)^{\#}$ with continuity.

Proof. Let $f \in X^{\#}$ and define $\gamma(g)=f * g(1)$. One has that $\gamma \in\left(X_{\mathcal{P}}\right)^{\prime}$ and $\|\gamma\| \leq\|f\|_{X^{\#}}$ what shows $X^{\#} \subset\left(X_{\mathcal{P}}\right)^{\prime}$.

Given $\gamma \in\left(X_{\mathcal{P}}\right)^{\prime}$ define $\lambda(z)=\sum_{n=0}^{\infty} \gamma\left(e_{n}\right) z^{n}$. Let $f \in X_{\mathcal{P}}$ and observe that from Proposition 4.1 (ii) the function $w \rightarrow f_{w}$ belongs to $A(\mathbb{D}, X)$. Hence

$$
\lambda * f(w)=\sum_{n=0}^{\infty} \gamma\left(e_{n}\right) \hat{f}(n) w^{n}=\gamma\left(f_{w}\right) .
$$

The continuity of $\gamma$ implies that $\lambda * f \in A(\mathbb{D})$. Moreover

$$
\|\lambda * f\|_{A(\mathbb{D})}=\sup _{|w|<1}|\lambda * f(w)| \leq K\|\gamma\|\|f\| .
$$

This shows that $\lambda \in\left(X_{\mathcal{P}}\right)^{\#}$ and $\|\lambda\|_{\left(X_{\mathcal{P}}\right)} \leq K\|\gamma\|$.

Corollary 4.2. If $X$ is an homogeneous Banach space then $X^{*}=\left(X_{\mathcal{P}}\right)^{*}=$ $\left(X_{\mathcal{P}}\right)^{\#}=\left(X_{\mathcal{P}}\right)^{\prime}$ with equivalent norms.

Proof. Since $X \subset \tilde{X}$ it follows from Proposition 3.3 that

$$
\tilde{X}_{\mathcal{P}}=X_{\mathcal{P}} \text { and } X^{*}=\left(X_{\mathcal{P}}\right)^{\#} .
$$

For the other equalities use the previous proposition.

Proposition 4.6. Let $X$ be homogeneous. Then $X_{\mathcal{P}} \subset X^{* *}$ and there exists $A>0$ that

$$
\|f\|_{X^{* *}} \leq\|f\|_{X} \leq K\|f\|_{X^{* *}}, \quad f \in X_{\mathcal{P}} .
$$

In particular, $X_{\mathcal{P}}=\left(X^{* *}\right)_{\mathcal{P}}$.

Proof. The inclusion and the first inequality are straightforward.

Let now $f \in X_{\mathcal{P}}$. From Corollary 4.2 and Hanh-Banach theorem,

$$
\begin{aligned}
\|f\|_{X} & =\sup \left\{|\gamma(f)|: \gamma \in\left(X_{\mathcal{P}}\right)^{\prime},\|\gamma\| \leq 1\right\} \\
& \leq A \sup \left\{|g * f(1)|: g \in\left(X_{\mathcal{P}}\right)^{\#},\|g\|_{\left(X_{\mathcal{P}}\right)} \leq 1\right\} \\
& \leq A \sup \left\{\|g * f\|_{\infty}: g \in\left(X_{\mathcal{P}}\right)^{\#},\|g\|_{\left(X_{\mathcal{P}}\right)} \leq 1\right\} \\
& =A \sup \left\{\|g * f\|_{\infty}: g \in X^{*},\|g\|_{X^{*}} \leq 1\right\} \leq A\|f\|_{X^{* *}} .
\end{aligned}
$$




\section{The Fatou property}

In this section we shall now consider a property closely related to (P7).

Definition 5.1. Let $X \subset \mathcal{H}(\mathbb{D})$ be an homogeneous Banach space. $X$ is said to satisfy F-property, to be denoted $(F P)$, if there exists $A>0$ such that for any sequence $\left(f_{n}\right) \in X$ with $\sup _{n}\left\|f_{n}\right\|_{X} \leq 1$ and $f_{n} \rightarrow f$ in $\mathcal{H}(\mathbb{D})$ one has that $f \in X$ and $\|f\|_{X} \leq A$.

Proposition 5.1. Let $X$ and $Y$ be $\mathcal{H}$-admissible Banach spaces. Then

(i) $\tilde{X}$ and $\mathfrak{B}^{X, q}, 1 \leq q \leq \infty$, have $(F P)$.

(ii) If $Y$ is homogeneous with $(F P)$ then $(X, Y)$ has $(F P)$.

Proof. (i) Let $\left(f_{n}\right) \in \tilde{X}$ such that $\left\|f_{n}\right\|_{\tilde{X}} \leq 1$ and $f_{n} \rightarrow f$ in $\mathcal{H}(\mathbb{D})$. Using that $\lim _{n \rightarrow \infty} M_{X}\left(r, f_{n}\right)=M_{X}(r, f)$ one concludes that $f \in \tilde{X}$. Similar argument works for $\mathfrak{B}^{X, q}$.

(ii) Let $\left(f_{n}\right) \in(X, Y)$ such that $\left\|f_{n}\right\|_{(X, Y)} \leq 1$ and $f_{n} \rightarrow f$ in $\mathcal{H}(\mathbb{D})$. Hence for a given $g \in X$ with $\|g\|_{X}=1$ we have $\left(f_{n} * g\right) \in Y$ such that $\left\|f_{n} * g\right\|_{(X, Y)} \leq 1$ and $f_{n} * g \rightarrow f * g$ in $\mathcal{H}(\mathbb{D})$. Since $Y$ has $(F P)$, one has that $f * g \in Y$ and $\|f * g\|_{Y} \leq A$. Therefore $f \in(X, Y)$ with $\|f\|_{(X, Y)} \leq A$.

Let us formulate some equivalent conditions of this property.

Theorem 5.1. Let $X$ be homogeneous. The following are equivalent:

(i) $X$ has $(F P)$.

(ii) If $f \in \mathcal{H}(\mathbb{D})$ and $\sup _{w \in \mathbb{D}}\left\|f_{w}\right\|_{X}<\infty$ then $f \in X$.

(iii) $X=\tilde{X}$ with equivalent norms.

(iv) $X=X^{* *}$.

Proof. (i) $\Longrightarrow$ (ii) Take $f \in \mathcal{H}(\mathbb{D})$ with $0<\sup _{0 \leq r<1} M_{X}(r, f)=A<\infty$. Select a sequence $r_{n}$ converging to 1 and put $f_{n}=A_{n} f_{r_{n}}$ where $A_{n}^{-1}=$ $M_{X}\left(r_{n}, f\right)$. Of course $f_{n} \rightarrow A^{-1} f$ in $\mathcal{H}(\mathbb{D})$ and $\left\|f_{n}\right\|_{X} \leq 1$. Applying the assumption one gets that $f \in X$.

(ii) $\Longrightarrow$ (iii) Note that if $X$ is homogeneous one has $X \subset \tilde{X}$ and $\|f\|_{\tilde{X}} \leq$ $K\|f\|_{X}$. The assumption means that $\tilde{X} \subset X$. The continuity follows from the open map theorem.

(iii) $\Longrightarrow$ (iv) Take $f \in X^{* *}$. Then $f_{r} \in\left(X^{* *}\right)_{\mathcal{P}}$ which, according to Proposition 4.6, coincides with $X_{\mathcal{P}}$. Hence we have

$$
M_{X}(r, f) \leq K M_{\left(X_{\mathcal{P}}\right)^{* *}}(r, f) \leq K^{\prime}\|f\|_{\left(X_{\mathcal{P}}\right)^{* *}} .
$$

This gives $f \in \tilde{X}=X$.

(iv) $\Longrightarrow$ (i) If $X=X^{* *}$ then $X$ has $(F P)$ because $\left(X^{*}, H^{\infty}\right)$ has $(F P)$ according to Proposition 5.1. 
This characterization allows us to give examples failing to have $(F P)$, for instance $X=c_{0}$ or $X=A(\mathbb{D})$.

To see that it suffices to consider the Cauchy kernel $C=(\hat{f}(j))_{j}$ where $\hat{f}(j)=1$ for all $j$. Hence $C \in \ell^{\infty} \backslash c_{0}$, but, however, $C_{w} * f=C_{w} \in c_{0}$ for any $|w|<1$ and $\sup _{w \in X}\left\|C_{w} * f\right\|_{c_{0}}=1$. Thus $c_{0}$ fails $(F P)$. Select $f \in H^{\infty} \backslash A(\mathbb{D})$ and observe that $\sup _{w \in X}\left\|C_{w} * f\right\|_{A(\mathbb{D})}=\|f\|_{\infty}$. Thus $A(\mathbb{D})$ fails $(F P)$.

In fact both examples are particular cases of the following corollary.

Corollary 5.1. If $X_{\mathcal{P}}$ has $(F P)$, then $X=X_{\mathcal{P}}$.

Remark 5.1. There exists a notion closely related to $(F P)$ in Banach space theory. Recall that a complex Banach space $E$ is said to have the $A R N P$ if any bounded $E$-valued function has boundary limits a.e, i.e if $F: \mathbb{D} \rightarrow E$ is holomorphic and bounded then $\lim _{r \rightarrow 1} F\left(r e^{i \theta}\right)$ exists a.e. in $E$ (see $[8,9]$ ).

Since $F(w)=f_{w} \in H^{\infty}(\mathbb{D}, X)$, one sees that any homogeneous Banach space $X$ with the $A R N P$ satisfies $(F P)$ (note that $f_{e^{i \theta}} \in X$ for almost all $\theta$ implies that $f \in X$.)

Since $H^{\infty}$ fails $A R N P$ but has $(F P)$ they are not equivalent properties.

Although the space $X \otimes Y$ needs not to have $(F P)$ if only one of the spaces has $(F P)$ (take $X=\ell^{\infty}$ and $Y=c_{0}$ and note that $X \otimes Y=c_{0}$ ) the following result says that the result holds true if both spaces have $(F P)$.

Theorem 5.2. Let $X$ and $Y$ be homogeneous with $(F P)$. Then $X \otimes Y$ has $(F P)$.

Proof. Let $\left(h_{n}\right) \in X \otimes Y$ such that $\left\|h_{n}\right\|_{X \otimes Y} \leq 1$ for all $n$ such that $h_{n} \rightarrow h$ in $\mathcal{H}(\mathbb{D})$. Let us take a decomposition such that $h_{n}=\sum_{j=1}^{\infty} f_{n, j} * g_{n, j}$ where $\left\|f_{n, j}\right\|_{X}=\left\|g_{n, j}\right\|_{Y}$ and

$$
\left\|h_{n}\right\|_{X \otimes Y} \leq \sum_{j=0}^{\infty}\left\|f_{n, j}\right\|_{X}\left\|g_{n, j}\right\|_{Y} \leq\left\|h_{n}\right\|_{X \otimes Y}+1 / n \leq 2 .
$$

Therefore for any sequence $\left(a_{j}\right)_{j} \in \ell^{2}$ with $\left\|\left(a_{j}\right)\right\|_{2}=1$ one has that

$$
\max \left\{\left\|\sum_{j} a_{j} f_{n, j}\right\|_{X},\left\|\sum_{j} a_{j} g_{n, j}\right\|_{Y}\right\} \leq 2 \text {. }
$$

Denoting $\phi_{n}=\sum_{j} a_{j} f_{n, j}$ and $\psi_{n}=\sum_{j} a_{j} g_{n, j}$, one has that $\sup _{n}\left\|\phi_{n}\right\|_{X} \leq 2$ and $\sup _{n}\left\|\psi_{n}\right\|_{X} \leq 2$. Since $X \subset\left(X^{\#}\right)^{\prime}$ and $Y \subset\left(Y^{\#}\right)^{\prime}$, the BanachAlaoglu theorem implies that there exists a subsequence $k(n)$ such that $\phi_{k(n)}$ converges in the weak ${ }^{*}$-topology to $\phi$ and $\psi_{k(n)}$ converges in the weak $k^{*}$ topology to $\psi$. In particular $\phi_{k(n)} \rightarrow \phi$ in $\mathcal{H}(\mathbb{D})$ and $\psi_{k(n)} \rightarrow \psi$ in $\mathcal{H}(\mathbb{D})$. Using the $(F P)$ in both spaces $X$ and $Y$ one obtains that $\phi \in X$ and $\psi \in Y$ with $\|\phi\|_{X} \leq 2$ and $\|\psi\|_{Y} \leq 2$. 
Let us now select $\left(a_{j}\right)_{j}$ the canonical basis of $\ell^{2}$ and write $f_{j}$ and $g_{j}$ the functions $\phi$ and $\psi$ corresponding to such cases. In particular, using a diagonal process there exists a subsequence $k^{\prime}(n)$ such that $f_{k^{\prime}(n), j} \rightarrow f_{j}$ and $g_{k^{\prime}(n), j} \rightarrow g_{j}$ in $\mathcal{H}(\mathbb{D})$ for all $j \in \mathbb{N}$. Taking limits one gets $f=\sum_{j=1}^{\infty} f_{j} * g_{j}$ in $\mathcal{S}$. To show that $\sum_{j}\left\|f_{j}\right\|_{X}\left\|g_{j}\right\|_{Y}<\infty$ we shall see that $\sum_{j}\left\|f_{j}\right\|_{X}^{2}<\infty$ and $\sum_{j}\left\|g_{j}\right\|_{Y}^{2}<\infty$. This follows using that $\phi=\phi\left(\left(a_{j}\right)\right)$ and $\psi=\psi\left(\left(a_{j}\right)\right)$ coincide with $\phi=\sum_{j} a_{j} f_{j}$ and $\psi=\sum_{j} a_{j} g_{j}$ and the facts $\left\|\sum_{j} a_{j} f_{j}\right\|_{X} \leq 2$ and $\left\|\sum_{j} a_{j} g_{j}\right\|_{Y} \leq 2$.

Theorem 5.3. Let $X$ and $Y$ be homogeneous spaces.

(i) If $Y$ has $(F P)$, then $(X, Y)=\left(X \otimes Y^{*}\right)^{*}$.

(ii) If $X$ and $Y$ have $(F P)$, then $X \otimes Y=\left(X, Y^{*}\right)^{*}$.

Proof. (i) Use that $Y^{* *}=Y$ and Corollary 2.2 to get $\left(X \otimes Y^{*}\right)^{*}=(X, Y)$.

(ii) We have $(X \otimes Y)^{* *}=X \otimes Y$ by Theorems 5.2 and 5.1. Again use $(X \otimes Y)^{*}=\left(X, Y^{*}\right)$ to conclude the proof.

\section{6. $\ell^{\infty} \otimes Y$ and solid Banach spaces}

Definition 6.1. (see [2]) $A$ set $A \subset \mathcal{S}$ is said to be solid if for any $f \in A$ and $g \in \mathcal{S}$ with $|\hat{g}(j)| \leq|\hat{f}(j)|, j \geq 0$, implies that $g \in A$.

Remark 6.1. Let $X$ be an $\mathcal{S}$-admissible Banach space. $X$ is solid iff $\ell^{\infty} \subset$ $(X, X)$.

Let us mention the following elementary facts.

Proposition 6.1. If $X$ or $Y$ are solid $\mathcal{S}$-admissible Banach spaces, then so are $(X, Y)$ and $X \otimes Y$.

Proof. Let $(\hat{f}(j))_{j} \in \ell^{\infty}$ and $\lambda \in(X, Y)$. To show that $f * \lambda \in(X, Y)$ take $g \in X$ and observe that $(f * \lambda) * g=\lambda *(f * g)=f *(\lambda * g)$. This shows that $(f * \lambda) * g \in Y$ whenever $X$ or $Y$ are solid.

The case $X \otimes Y$ follows from Remark 6.1 together with the trivial inclusion $X \subset(Y, X \otimes Y)$ and Theorem 2.3. If $X$ is solid then

$$
\ell^{\infty} \subset(X, X) \subset(X,(Y, X \otimes Y)=(X \otimes Y, X \otimes Y) .
$$

Proposition 6.2. (see [2]) If $X \subset \mathcal{S}$ is an $\mathcal{S}$-admissible Banach space, then there is a largest solid $\mathcal{S}$-admissible Banach space $s(X) \subset X$. Furthermore $s(X)$ is the largest solid subset of $X$ and we have

$$
s(X)=\left(\ell^{\infty}, X\right) .
$$


Proof. Denote $s(X)=\left(\ell^{\infty}, X\right)$. It is an $\mathcal{S}$-admissible Banach space, by Theorem 2.1. From Proposition 6.1 one has that $s(X)$ is a solid subspace of $X$. Now let $Y \subset X$ be any other solid subset. If $f \in Y$ and $g \in \ell^{\infty}$, then $g * f \in Y \subset X$. Hence $f \in\left(\ell^{\infty}, X\right)$ and so $Y \subset\left(\ell^{\infty}, X\right)$.

Proposition 6.3. ([2,7]) If $X \subset \mathcal{S}$, then there is a smallest solid superset $S(X) \supset X$. Furthermore,

$$
\begin{gathered}
S(X)=\ell^{\infty} * X, \quad \text { and } \\
S(X)=\{g \in \mathcal{S}: \exists f \in A \text { such that }|\hat{f}(j)| \geq|\hat{g}(j)| \text { for all } j\} .
\end{gathered}
$$

Proof. Clearly, $S(X)$ is the intersection of all solid sets containing $X$. Since the set $\ell^{\infty} * X$ is solid, we have $S(X) \subset \ell^{\infty} * X$. On the other hand,

$$
\left.\ell^{\infty} * X \subset \ell^{\infty} * S(X) \quad \text { (because } X \subset S(X)\right)
$$

and $\ell^{\infty} * S(X)=S(X)$, whence $\ell^{\infty} * X \subset S(X)$, and so $\ell^{\infty} * X=S(X)$.

For $(\dagger)$, let

$$
B=\{g \in \mathcal{S}: \exists f \in X \text { such that }|\hat{f}(j)| \geq|\hat{g}(j)| \text { for all } j\} .
$$

It is trivial to check that $B$ is a solid superset of $X$. Let $D$ be any solid superspace of $A$, and let $g \in B$. Then there is $f \in X$ such that $|\hat{f}(j)| \geq|\hat{g}(j)|$ for all $j$. Then $f \in D$, and since $D$ is solid we have $g \in D$. Thus $B \subset D$, whence $B=S(X)$.

Denote $S_{b}(X)=\ell^{\infty} \otimes X$. Of course $S(X) \subset S_{b}(X)$.

Theorem 6.1. Let $X$ be an $\mathcal{S}$-admissible Banach space. Then $S_{b}(X)$ is the smallest solid Banach space containing $X$. More precisely, if $Y$ is a solid Banach space containing $X$, then $S_{b}(X) \subset Y$ with continuity.

Proof. Let $h \in \ell^{\infty} \otimes X$. Then

$h=\sum_{n=1}^{\infty} b_{n} * f_{n}$, where $b_{n} \in \ell^{\infty}, f_{n} \in X$, and $\left\|b_{n}\right\|_{\ell^{\infty}}=1, \sum_{n=1}^{\infty}\left\|f_{n}\right\|_{X}<\infty$.

The series $\sum_{n=1}^{\infty} b_{n} * f_{n}$ converges in $Y$ because

$$
\sum_{n=1}^{\infty}\left\|b_{n} * f_{n}\right\|_{Y} \leq \sum_{n=1}^{\infty} C\left\|f_{n}\right\|_{Y} \leq \sum_{n=1}^{\infty} C C_{1}\left\|f_{n}\right\|_{X}<\infty
$$

The sum in $Y$ of this series is equal to $h$ because $X$ and $Y$ are continuously embedded in $\mathcal{S}$. Thus $h \in Y$, which was to be proved. 
Corollary 6.1. If $S(X)$ is an $\mathcal{S}$-admissible Banach space then $S(X)=$ $\ell^{\infty} \otimes X$, with equivalent norms.

Proof. Since $S(X) \subset \ell^{\infty} \otimes X$, by definition, and $\ell^{\infty} \otimes X \subset S(X)$, by Theorem 6.1 , we see that $S(X)$ and $\ell^{\infty} \otimes X$ are equal as sets. The norms are equivalent because these spaces are complete and $\ell^{\infty} \otimes X \subset S(X)$, by Theorem 6.1.

Theorem 6.2. If $X$ and $Y$ are $\mathcal{S}$-admissible Banach spaces, then

$$
\left(S_{b}(X), Y\right)=(X, s(Y))=s((X, Y)) .
$$

Proof. We have, by Theorem 2.3,

$$
\left(\ell^{\infty} \otimes X, Y\right)=\left(\ell^{\infty},(X, Y)\right)=s((X, Y)),
$$

and

$$
\left(X \otimes \ell^{\infty}, Y\right)=\left(X,\left(\ell^{\infty}, Y\right)\right)=(X, s(Y))
$$

\section{Computing $H^{1} \otimes X$ in some cases}

The aim of this section is to identify $H^{1} \otimes X$ for some homogeneous Banach spaces $X$. According to Theorem 5.3 one can state the following general result.

Proposition 7.1. If $X$ has (FP) then we have that

$$
H^{1} \otimes X=\left(H^{1}, X^{*}\right)^{*}=(X, B M O A)^{*} .
$$

However this is not a direct description of the space, but relies upon the knowledge of the multiplier space. The following lemma is relevant for our purposes.

Lemma 7.1. Let $X$ be a homogeneous Banach space. Then there exist $A_{1}, A_{2}>0$ such that

$$
A_{1} r^{m}\|f\|_{X} \leq M_{X}(r, f) \leq A_{2} r^{k}\|f\|_{X}, \quad 0<r<1
$$

whenever $f(z)=\sum_{j=k}^{m} a_{j} z^{j}$ where $0 \leq k<m$.

Proof. It is well known (see Lemma 3.1 [21]) that

$$
r^{m}\|f\|_{\infty} \leq M_{\infty}(r, f) \leq r^{k}\|f\|_{\infty}, \quad 0<r<1 .
$$

Using Proposition 4.6 one has

$$
\begin{aligned}
r^{m}\|f\|_{X} & \approx r^{m}\|f\|_{X^{* *}} \approx \sup \left\{r^{m}\|f * g\|_{\infty}:\|g\|_{X^{*}}=1\right\} \\
& \leq C \sup \left\{M_{\infty}(r, f * g):\|g\|_{X^{*}}=1\right\} \approx\left\|f_{r}\right\|_{X^{* *}} \approx M_{X}(r, f) \\
& \leq C r^{n} \sup \left\{\|f * g\|_{\infty}:\|g\|_{X^{*}}=1\right\} \leq C r^{n}\|f\|_{X^{* *}} \leq A r^{n}\|f\|_{X} .
\end{aligned}
$$


Lemma 7.2. Let $X \subset \mathcal{H}(\mathbb{D})$ be homogeneous and

$$
P(z)=\sum_{k=2^{n-1}}^{2^{n+1}} \hat{P}(k) e_{k} .
$$

Then there exist constants $B_{1}$ and $B_{2}$ such that

$$
B_{1} 2^{n}\|P * f\|_{X} \leq\|P * D f\|_{X} \leq B_{2} 2^{n}\|P * f\|_{X}, f \in X
$$

Proof. We apply Lemma 7.1 to obtain

$$
A_{1} r^{2^{n+1}}\|P\|_{X} \leq M_{X}(r, P) \leq A_{2} r^{2^{n-1}}\|P\|_{X} .
$$

To show (7.1) apply (7.2) for $r_{n}=1-2^{-n}$ and (3.1) to get first

$$
\begin{aligned}
\|P * D f\|_{X} & =\|D(P * f)\|_{X} \leq A M_{X}\left(r_{n}, D(P * f)\right) \\
& \leq A 2^{n} M_{X}\left(r_{n}, P * f\right) d s \leq A 2^{n}\|P * f\|_{X} .
\end{aligned}
$$

Also applying (3.2) one gets

$$
\begin{aligned}
\|P * f\|_{X} & \approx M_{X}\left(r_{n}, P * f\right) \leq A \int_{0}^{r_{n}} M_{X}(s, P * D f) d s \\
& \leq A \int_{0}^{r_{n}} s^{2^{n}}\|P * D f\|_{X} d s \leq A 2^{-n}\|P * D f\|_{X} .
\end{aligned}
$$

Theorem 7.1. Let $X$ be an homogeneous Banach space. Then

$$
\mathfrak{B}^{X, 1} \subset H^{1} \otimes X \subset X_{\mathcal{P}} .
$$

Proof. From Proposition 2.2 it suffices to show that if $f \in H^{1}$ and $g \in X$ then $f * g \in X_{\mathcal{P}}$. From Lemma 3.1

$$
M_{X}\left(r^{2}, f * g\right) \leq M_{1}(r, f) M_{X}(r, g) \leq K\|f\|_{1}\|g\|_{X} .
$$

Using Proposition 2.1 the polynomials are dense in $H^{1} \otimes X$ and $H^{1} \otimes X \subset X_{p}$ is shown.

Let us now show that $\mathfrak{B}^{X, 1} \subset H^{1} \otimes X$.

Let $\left\{W_{n}\right\}_{0}^{\infty}$ be a sequence of polynomials such that

$$
\begin{gathered}
\operatorname{supp}\left(\hat{W}_{n}\right) \subset\left[2^{n-1}, 2^{n+1}\right] \quad(n \geq 1), \quad \operatorname{supp}\left(\hat{W}_{0}\right) \subset[0,1], \quad \sup _{n}\left\|W_{n}\right\|_{1}<\infty \\
f=\sum_{n=0}^{\infty} W_{n} * f, \quad f \in \mathcal{H}(\mathbb{D}) .
\end{gathered}
$$


Such a sequence exists (see, e.g., [4, 23, 17, 25] for possible constructions). Note that

$$
\left\|\left(W_{n} * f\right)_{r}\right\|_{X} \leq K\left\|W_{n}\right\|_{1}\left\|f_{r}\right\|_{X} \leq C\|f\|_{X},
$$

Hence, since $W_{n} * f$ is a polynomial, $\left\|W_{n} * f\right\|_{X} \leq C\|f\|_{X}$.

Denoting $Q_{n}=W_{n-1}+W_{n}+W_{n+1}$ we can write

$$
f=\sum_{n=0}^{\infty} Q_{n} * W_{n} * f,
$$

for all $f \in \mathcal{H}(\mathbb{D})$. Note now that Lemma 7.2 allow us to conclude

$$
\begin{aligned}
\sum_{n=0}^{\infty}\left\|Q_{n}\right\|_{1}\left\|W_{n} * f\right\|_{X} & \leq K \sum_{n=0}^{\infty}\left\|W_{n} * f\right\|_{X} \\
& \leq K \sum_{n=0}^{\infty} \int_{1-2^{-n}}^{1-2^{-(n+1)}} 2^{n} r^{2^{n}}\left\|W_{n} * f\right\|_{X} d r \\
& \leq K \sum_{n=0}^{\infty} \int_{1-2^{-n}}^{1-2^{-(n+1)}} r^{2^{n}}\left\|W_{n} * D f\right\|_{X} d r \\
& \leq K \sum_{n=0}^{\infty} \int_{1-2^{-n}}^{1-2^{-(n+1)}} M_{X}\left(r, W_{n} * D f\right) d r \\
& \leq K \sum_{n=0}^{\infty} \int_{1-2^{-n}}^{1-2^{-(n+1)}} M_{X}(r, D f) d r \\
& =K \int_{0}^{1} M_{X}(r, D f) d r=K\|f\|_{\mathfrak{B}^{X}, 1} .
\end{aligned}
$$

A property that turns out to be crucial for our purposes is the following one already mentioned in the introduction.

Definition 7.1. Let $X \subset \mathcal{H}(\mathbb{D})$ be an homogeneous Banach space. We say that $X$ satisfies $(H L P)$ if $X \subset \mathfrak{B}^{X, 2}$, i.e. there exits a constant $A>0$ such that

$$
\int_{0}^{1}(1-r) M_{X}^{2}(r, D f) d r \leq A\|f\|_{X}
$$

Theorem 7.2. Let $X$ be an homogeneous Banach space satisfying $(H L P)$. Then $H^{1} \otimes X=\mathfrak{B}^{X, 1}$. 
Proof. Due to Theorem 7.1 we only need to show that $H^{1} \otimes X \subset \mathfrak{B}^{X, 1}$. It suffices to see that $f * g \in \mathfrak{B}^{X, 1}$ for each $f \in H^{1}$ and $g \in X$. Now using Lemma 3.1 we have,

$$
\begin{aligned}
\int_{0}^{1} M_{X}(r, D(f * g)) r d r & \leq A \int_{0}^{1}\left(\int_{0}^{r} M_{X}\left(s, D^{2}(f * g)\right) d s\right) r d r \\
& \leq A \int_{0}^{1}(1-s) M_{X}\left(s, D^{2}(f * g)\right) d s \\
& \leq 2 A\left(\int_{0}^{1}\left(1-r^{2}\right) M_{1}(r, D f) M_{X}(r, D g) r d r\right.
\end{aligned}
$$

Now from Cauchy-Schwarz (7.3) for $\mathbb{C}$-valued functions and (HLP) one obtains

$$
\begin{aligned}
\int_{0}^{1}(1 & \left.-r^{2}\right) M_{1}(r, D f) M_{X}(r, D f) r d r \leq \\
& \leq\left(\int_{0}^{1}\left(1-r^{2}\right) M_{1}^{2}(r, D f) r d r\right)^{1 / 2}\left(\int_{0}^{1}\left(1-r^{2}\right) M_{X}^{2}(r, D g) r d r\right)^{1 / 2} \\
& \leq K\|f\|_{1}\|g\|_{X}
\end{aligned}
$$

\section{Applications}

Our techniques allow us to describe $X \otimes Y$ in several cases. We only exhibit some applications, although many others can be achieved in a similar fashion.

As a consequence of Theorem 7.2 and Proposition 3.6 one obtains the following result.

Corollary 8.1. Let $1 \leq p \leq 2$. Then

(i) $H^{1} \otimes H^{p}=\mathfrak{B}^{p, 1}$.

(ii) $H^{1} \otimes \ell^{p}=\ell^{p, 1}$.

Let $1 \leq p, q \leq \infty$ and let $H^{p, q, \alpha}$ denote the mixed norm spaces of analytic functions in the unit disc given by the condition

$$
\|f\|_{H^{p, q, \alpha}}=\left(\int_{0}^{1}(1-r)^{\alpha q-1} M_{p}^{q}(r, f) d r\right)^{1 / q}<\infty, \quad q<\infty
$$

and

$$
\|f\|_{H^{p, \infty, \alpha}}=\sup _{0<r<1}(1-r)^{\alpha} M_{p}(r, f)<\infty, \quad q=\infty .
$$

Recall that $p \ominus q$ stands for the value $\infty$ whenever $q \geq p$ and $\frac{1}{p \ominus q}=\frac{1}{q}-\frac{1}{p}$ whenever $q<p$, and that $\frac{1}{p * q}=\min \left\{1, \frac{1}{p}+\frac{1}{q}\right\}$. 
Corollary 8.2. Let $1 \leq q, u, v \leq \infty$. Then $\mathfrak{B}^{1, q} \otimes \mathfrak{B}^{u, v}=\mathfrak{B}^{u, q * v}$.

Proof. This follows from Theorem 5.3, applying that the spaces $\mathfrak{B}^{p, q}$ have $(F P)$ together with the facts that

$$
\left(\mathfrak{B}^{p, q}, H^{\infty}\right)=\mathfrak{B}^{p^{\prime}, q^{\prime}}, \quad p, q \geq 1,
$$

(see [1] for $p=1,1<q<\infty$; see [13] for the remaining cases) and

$$
\left(\mathfrak{B}^{1, q}, \mathfrak{B}^{u^{\prime}, v^{\prime}}\right)=\mathfrak{B}^{u^{\prime}, q \ominus v^{\prime}}, \quad q, u, v \geq 1 .
$$

Relation (8.1) is only a reformulation the following result on multipliers (see [17, Theorem 3.5]):

$$
\left(H(1, q, 1), H\left(u^{\prime}, v^{\prime}, 1\right)\right)=\left\{\lambda \in \mathcal{H}(\mathbb{D}): D \lambda \in H\left(u^{\prime}, q \ominus v^{\prime}, 1\right)\right\} .
$$

We can now use our techniques to characterize the space of multipliers from $H^{1}$ in some cases.

Theorem 8.1. Let $X$ be a homogeneous Banach space with $(H L P)$. Then

$$
\begin{aligned}
\left(H^{1}, X^{*}\right) & =\mathfrak{B}^{X^{*}, \infty} . \\
\left(H^{1}, X^{K}\right) & =\mathfrak{B}^{X^{K}, \infty} .
\end{aligned}
$$

Proof. Apply Theorem 2.3 together with Theorem 7.2 and Proposition 4.4 to obtain

$$
\left(H^{1}, X^{*}\right)=\left(H^{1} \otimes X, H^{\infty}\right)=\left(\mathfrak{B}^{X, 1}, H^{\infty}\right)=\mathfrak{B}^{X^{*}, \infty} .
$$

The other case is analogous.

In particular the previous theorem yields the following results on multipliers from $H^{1}$ due, among others, to Hardy and Littlewood, Stein and Zygmund, Sledd (the cases $H^{q}$ ), to Mateljević and Pavlović (the case BMOA) and to Duren (the case $\ell^{q}$ ).

Corollary 8.3. Let $2 \leq q<\infty$. Then

$$
\begin{gathered}
\left(H^{1}, H^{q}\right)=\mathfrak{B}^{q, \infty} \quad(\text { see }[16],[28],[27]), \\
\left(H^{1}, B M O A\right)=\mathfrak{B} \quad(\text { see }[22]), \\
\left(H^{1}, \ell^{q}\right)=\ell(q, \infty) .
\end{gathered}
$$

Also we can use our results to obtain spaces of multipliers into $B M O A$ in some cases. 
Theorem 8.2. Let $X$ be a homogeneous Banach space with (HLP). Then

$$
(X, B M O A)=\mathfrak{B}^{X^{*}, \infty} .
$$

Proof. Combining again Theorem 2.3 together with Theorem 7.2 and Proposition 4.4 one gets

$$
(X, B M O A)=\left(X,\left(H^{1}, H^{\infty}\right)\right)=\left(X \otimes H^{1}, H^{\infty}\right)=\left(\mathfrak{B}^{X, 1}, H^{\infty}\right)=\mathfrak{B}^{X^{*}, \infty} .
$$

Corollary 8.4. Let $1 \leq p \leq 2$. Then

$$
\begin{gathered}
\left(H^{p}, B M O A\right)=\mathfrak{B}^{p^{\prime}, \infty} \quad(\text { see }[24] \text { and }[17]), \\
\left(\ell^{p}, B M O A\right)=\ell\left(p^{\prime}, \infty\right) .
\end{gathered}
$$

The results allow also to recapture some of the multiplier results for Hardy-Lorentz spaces appearing in [19] using similar approaches.

\section{References}

[1] Ahern, P. And Jevtić, M.: Duality and multipliers for mixed norm spaces. Michigan Math. J. 30 (1983), no. 1, 53-64.

[2] Anderson, J. M. And Shields, A. L.: Coefficient multipliers of Bloch functions. Trans. Amer. Math. Soc. 224 (1976), no. 2, 255-265.

[3] Arazy, J., Fisher, S. And Peetre, J.: Möbius invariant function spaces. J. Reine Angew. Math. 363 (1985), 110-145.

[4] Blasco, O.: Multipliers on spaces of analytic functions. Canad. J. Math. 47 (1995), no. 1, 44-64.

[5] Blasco, O.: Vector-valued analytic functions of bounded mean oscillation and geometry of Banach spaces. Illinois J. Math. 41 (1997), 532-558.

[6] Blasco, O.: Composition operators on the minimal space invariant under Moebious transformations. In Complex and harmonic analysis, 157-166. DEStech Publ., Lancaster, PA, 2007.

[7] Buckley, S. M.: Relative solidity for spaces of holomorphic functions. Math. Proc. R. Ir. Acad. 104A (2004), no. 1, 83-97 (electronic).

[8] Bukhvalov, A. V.: On the analytic Radon-Nikodým property. In Function Spaces (Poznań 1989), 211-228. Teubner-Texte Math 120. Teubner, Stuttgart, 1991.

[9] Bukhvalov, A. V. And Danilevich, A. A.: Boundary properties of analytic functions with values in Banach spaces. Mat. Zametki 31 (1982), 203-214 (russian).

[10] Duren, P. L.: Theory of $H^{p}$-spaces. Pure and Applied Mathematics 38. Academic Press, New York-London, 1970. 
[11] Figà-Talamanca, A.: Translation invariant operators in $L^{p}$. Duke Math. J. 32 (1965), 495-501.

[12] Fisher, S.: The Möbius group and invariant spaces of analytic functions. Amer. Math. Monthly 95 (1988), 514-527.

[13] Flett, T. M.: Lipschitz spaces of functions on the circle and the disc. J. Math. Anal. Appl. 39 (1972), 125-158.

[14] Girela, D., Pavlović, M. And Peláez, J. A.: Spaces of analytic functions of Hardy-Bloch type. J. Anal. Math. 100 (2006), 53-81.

[15] Hardy, G. H. and Littlewood, J. E.: Notes on the theory of series. XX: Generalizations of a theorem of Paley. Q. J. Math., Oxford Ser. 8 (1937), $161-171$.

[16] Hardy, G. H. And Littlewood, J. E.: Theorems concerning mean values of analytic or harmonic functions Quart. J. Math., Oxford Ser. 12 (1941), 221-256.

[17] Jevtić, M. And Pavlović, M.: Coefficient multipliers on spaces of analytic functions. Acta Sci. Math. (Szeged) 64 (1998), no. 3-4, 531-545.

[18] KellogG, C. N.: An extension of the Hausdorff-Young theorem. Michigan Math. J. 18 (1971), 121-127.

[19] Lengfield, M.: A nested embedding theorem for Hardy-Lorentz spaces with applications to coefficient multiplier problems. Rocky Mountain J. Math. 38 (2008), no. 4, 1215-1251.

[20] Mateljević, M. And Pavlović, M.: $L^{p}$-behaviour of power series with positive coefficients and Hardy spaces. Proc. Amer. Math. Soc. 87 (1983), 309-316.

[21] Mateljević, M. And Pavlović, M.: $L^{p}$-behaviour of the integral means of analytic functions. Studia Math. 77 (1984), no. 3, 219-237.

[22] Mateljević, M. and Pavlović, M.: Multipliers of $H^{p}$ and BMOA. Pac. J. Math. 146 (1990), no. 1, 71-84.

[23] Mateljević, M. And Pavlović, M.: The best approximation and composition with inner functions. Michigan Math. J. 42 (1995), no. 2, 367-378.

[24] Nowak, M.: A note on coefficient multipliers $\left(H^{p}, \mathfrak{B}\right)$ and $\left(H^{p}, \mathrm{BMOA}\right)$. In Topics in complex analysis (Warsaw, 1992), 299-302. Banach Center Publ. 31. Polish Acad. Sci., Warsaw, 1995.

[25] Pavlović, M.: Mixed norm spaces of analytic and harmonic functions. I. Publ. Inst. Math. (Beograd) (N.S.) 40 (54) (1986), 117-141.

[26] Rubel, L. A. And Timoney, R. M.: An extremal property of the Bloch space. Proc. Amer. Math. Soc. 75 (1979), 45-49.

[27] Sledd, W. T.: On multipliers of $H^{p}$ spaces. Indiana Univ. Math. J. 27 (1978), no. 5, 797-803.

[28] Stein, E. M. And Zygmund, A.: Boundedness of translation invariant operators on Hölder spaces and $L^{p}$-spaces. Ann. of Math. (2) 85 (1967), $337-349$. 
[29] TAYlor, A. E.: Banach spaces of functions analytic in the unit circle. I. Studia Math. 11 (1950), 145-170.

[30] TAYlor, A. E.: Banach spaces of functions analytic in the unit circle. II. Studia Math. 12 (1951), 25-50.

[31] Timoney, R. M.: Natural function spaces. J. London Math. Soc. (2) 41 (1990), 78-88.

[32] Timoney, R. M.: Maximal invariant spaces of analytic functions. Indiana Univ. Math. J. 31 (1982), 651-663.

[33] Torchinsky, A.: Real-variable methods in harmonic analysis. (Reprint of the 1986 original). Dover Publications, Mineola, NY, 2004.

[34] Wells, J. H.: Some results concerning multipliers of $H^{p}$. J. London Math. Soc. (2) 2 (1970), 549-556.

Recibido: 29 de junio de 2009

Óscar Blasco

Departamento de Análisis Matemático Universidad de Valencia (Burjassot)

46100, Valencia, Spain oblasco@uv.es

Miroslav Pavlović

Matematički fakultet Studentski trg 16, 11001

Beograd, p.p. 550, Serbia

pavlovic@matf.bg.ac.rs

The first author is partially supported by Spanish project MTM2008-04594/MTM. The second author is supported by MNTR Srbija Project 0N174017. 